\title{
Modified viral-genetic mapping reveals local and global connectivity relationships of
} ventral tegmental area dopamine cells

\author{
Kevin T. Beier
}

6 Department of Physiology and Biophysics, Neurobiology and Behavior, Biomedical Engineering,

7 Pharmaceutical Sciences, Center for the Neurobiology of Learning and Memory, University of

8 California, Irvine, Irvine, CA, USA 92697

10 Correspondence: kbeier@uci.edu

\section{ABSTRACT}

15 Dopamine cells in the ventral tegmental area $\left(V T A^{\mathrm{DA}}\right)$ are critical for a variety of motivated behaviors. These cells receive synaptic inputs from over 100 anatomically-defined brain regions, which enables control from a distributed set of inputs across the brain. Extensive efforts have been made to map inputs to VTA cells based on neurochemical phenotype and output site. However, all of these studies have the same fundamental limitation that inputs local to the VTA cannot be properly assessed due to non-Cre-dependent uptake of EnvA-pseudotyped virus.

21 Therefore, the quantitative contribution of local inputs to the VTA, including GABAergic, DAergic,

22 and serotonergic, is not known. Here, we used a modified viral-genetic strategy that enables

23 examination of both local as well as long-range inputs to VTA ${ }^{\mathrm{DA}}$ cells. We found that nearly half 24 of the total inputs to VTA ${ }^{\mathrm{DA}}$ cells are located locally, revealing a substantial portion of inputs that 25 have been missed by previous analyses. The majority of inhibition to VTA ${ }^{\mathrm{DA}}$ cells arises from the 26 substantia nigra pars reticulata, with large contributions from the VTA and the substantia nigra 27 pars compacta. In addition to receiving inputs from VTA ${ }^{\text {GABA }}$ neurons, DA neurons are connected 28 with other DA neurons within the VTA as well as the nearby retrorubal field. Lastly, we show that $29 V_{T} A^{\mathrm{DA}}$ neurons receive inputs from distributed serotonergic neurons throughout the midbrain and 30 hindbrain, with the majority arising from the dorsal raphe. Our study highlights the importance of 31 using the appropriate combination of viral-genetic reagents to unmask the complexity of 32 connectivity relationships to defined cells in the brain. 


\section{INTRODUCTION}

36 VTA ${ }^{\text {DA }}$ cells mediate a variety of motivated behaviors, including reward and aversion.(Björklund

37 \& Dunnett, 2007; Bromberg-Martin et al., 2010; Cohen et al., 2012; Lammel et al., 2012; Wise,

38 2004). Substantial effort has been made to map the brain regions and cell types that provide input

39 to and receive projections from VTA ${ }^{\mathrm{DA}}$ cells, a critical step towards understanding how VTA ${ }^{\mathrm{DA}}$ cells

40 effect behavioral consequences in response to a variety of stimuli. The recent advent of one-step

41 rabies virus (RABV) has enabled the mapping of inputs onto defined cell types (Wickersham et

42 al., 2007). This strategy was employed nearly a decade ago to map brain-wide inputs to DA cells

43 in the VTA and the adjacent substantia nigra pars compacta (SNc) (Watabe-Uchida et al., 2012).

44 However, DA neurons in the VTA and SNc are not homogenous, but rather are heterogenous in 45 their molecular signatures, projection patterns, physiological properties, and behavioral functions 46 (Kim et al., 2016; Lammel et al., 2008, 2011, 2012; Lerner et al., 2015). We therefore designed a 47 method, Tracing the Relationship between Inputs and Outputs (TRIO) to map the input-output relationship of projection-defined VTA ${ }^{\mathrm{DA}}$ cells (Beier et al., 2015; Lerner et al., 2015; Schwarz et al., 2015). TRIO revealed that different subtypes of VTA ${ }^{\mathrm{DA}}$ cells received biased inputs, and that global input-output maps could be used to infer the behavioral contribution of particular input sites, such as the cortex (Beier et al., 2015).

While these and more recent studies have mapped global inputs to VTA ${ }^{\mathrm{DA}}$ and $\mathrm{SNc}{ }^{\mathrm{DA}}$ cells (Beier et al., 2015; Faget et al., 2016; Lerner et al., 2015; Menegas et al., 2015; Watabe-Uchida et al., 2012) each study has the same limitation in that only inputs located at a distance from the injection site in the midbrain can be assessed. This is because the Cre-dependent TVA protein that facilitates EnvA-mediated infection is also expressed at low levels in non-Cre-expressing cells near the site of injection (Miyamichi et al., 2013). Therefore, RABV virions that are pseudotyped with the EnvA protein can infect both Cre-expressing starter cells as well as non-Cre-expressing cells nearby that express low levels of TVA through leaky, non-Cre-dependent expression. This leaky gene expression is due to incomplete suppression of transcription/translation of TVA. While this may not be an issue with fluorescent molecules or chemogenetic effectors such as DREADDs (Botterill et al., 2021), in cases where only small amounts of a gene product are required to exert function, such as TVA, the problem becomes magnified. Only a single functional unit 65 presumably three TVA molecules bound to an EnvA trimer (Alsteens et al., 2017) - is required 66 to enable infection of EnvA-pseudotyped RABV. Injection of $A A V-C A G-F L E x^{10 x P}$-TVA and AAV67 CAG-FLEx ${ }^{\text {loxP }}-R A B V-G 2$ weeks prior to injection of EnvA-pseudotyped RABV can result in 68 thousands of infection events near the injection site, even in Cre- animals that should not express 
TVA (Beier et al., 2015). Notably, these infections do not occur if AAVs are not injected, demonstrating that the background is TVA-mediated. Therefore, whether RABV-labeled neurons near the injection site were bona-fide inputs to starter cells or cells that were infected via the viral inoculum in previous VTA ${ }^{\mathrm{DA}}$ mapping experiments cannot be distinguished.

One solution to this problem is to reduce the efficiency of TVA-mediated infection. A mutant version of TVA with a single point mutation ( $\mathrm{Glu}^{66} \rightarrow \mathrm{Thr}$, or $66 \mathrm{~T}$ ) that exhibits only $10 \%$ of the efficiency of the wild-type TVA dramatically reduces local background (Miyamichi et al., 2013; Rong et al., 1998). Using this variant enables analysis of the quantitative contribution of local inputs to defined cell types, such as VTA ${ }^{D A}$ neurons. Here, we quantified the inputs to VTA ${ }^{D A}$ neurons, comparing long-distance inputs to local inputs. We used the $T C^{66 T}$ variant in place of the wild type TVA, $T C^{B}$, in our tracing studies and found that almost half of the inputs to VTA ${ }^{\mathrm{DA}}$ cells are located near the VTA; many of these represent inputs that were missed or may have been mis-quantified in previous studies. We then performed quantitative analyses to identify the biases that local inputs have onto different sets of VTA ${ }^{\mathrm{DA}}$ cells. We then examined the sources of local GABAergic input to VTA ${ }^{\mathrm{DA}}$ cells, explored potential connections between DA cells in the ventral midbrain, and quantified the sources of serotonergic inputs to VTA ${ }^{D A}$ cells. Our analysis is the first of its kind to detail the quantitative contributions of inhibition and neuromodulatory influence from local cells in the midbrain and hindbrain onto VTA ${ }^{\text {DA }}$ cells, providing a comprehensive global picture of the main cell populations that influence and control VTA ${ }^{\mathrm{DA}}$ cells.

\section{RESULTS}

91 We performed RABV one-step mapping using $T C^{66 T}$ in place of $T C^{B}$ (Miyamichi et al., 2013)

92 (Figure 1A). We first injected a combination of Cre-dependent adeno-associated viruses (AAVs)

93 encoding the RABV glycoprotein, RABV-G, and $T C^{66 T}$ into the VTA of DAT-Cre mice that express

94 the Cre recombinase in DA neurons. Two weeks later, we injected EnvA-pseudotyped, G-deleted,

95 GFP-expressing RABV into the VTA. We then allowed 5 days for RABV spread to input cells

96 before terminating the experiment.

98 Control experiments in non-Cre-expressing mice were performed to examine the local 99 background of non-Cre-mediated, $T C^{66 T}$-facilitated infection. We previously observed using $T C^{B}$ 100 that, on average, 3,183 cells were infected in a non-Cre-dependent, $T C^{B}$-facilitated fashion per 101 mouse (Beier et al., 2015). Using $T C^{66 T}$, we found an average of 2.67 cells per brain 102 (Supplemental Figure 1). This background infection was indistinguishable from controls where no 

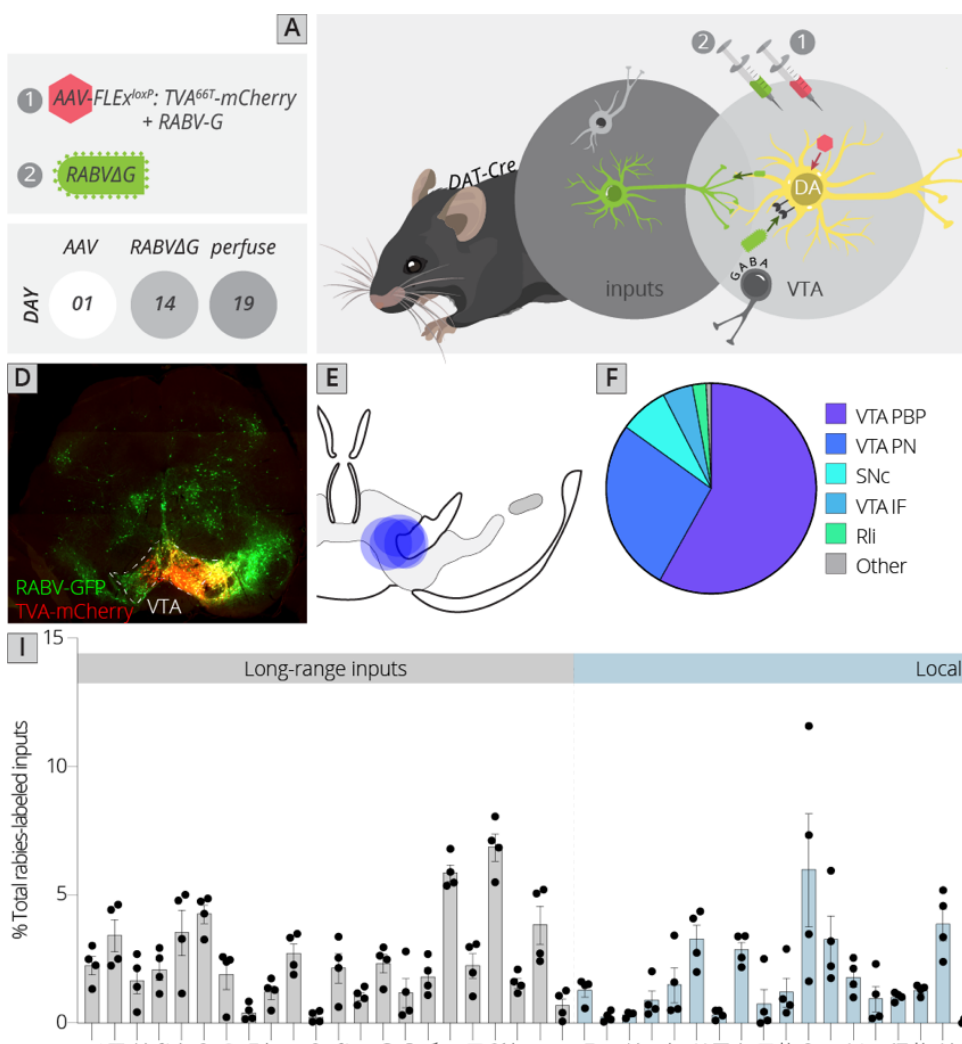

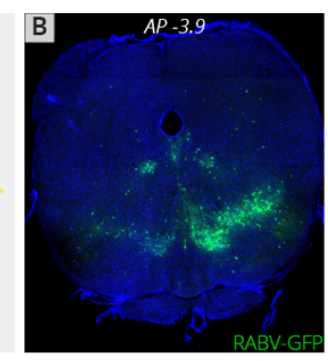

G
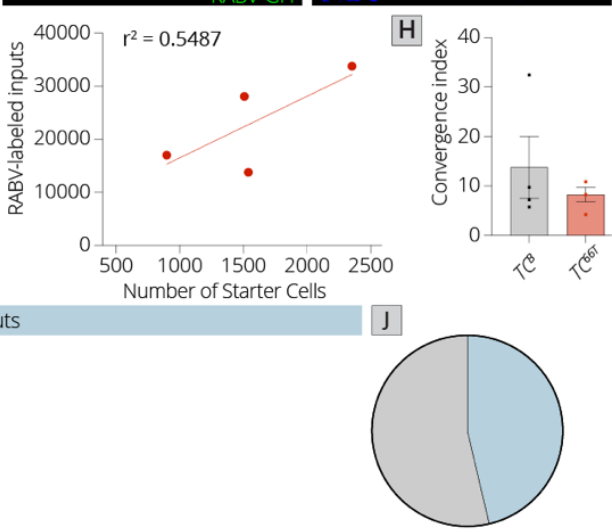

\% long distance $\square$ local
103

104

105

Figure 1: Use of a modified viral-genetic strategy to map local and global inputs to VTA ${ }^{\mathrm{DA}}$ neurons.

(A) Strategy for viral mapping. On day 1, a combination of AAVs expressing the mutated TVA protein fused to mCherry, $T C^{66 T}$, and RABV-G were injected into the VTA. Two weeks later, EnvApseudotyped RABV expressing GFP was injected into the VTA. Animals were sacrificed five days later.

(B) Representative image of local virally-labeled inputs at anterior-posterior $-3.9 \mathrm{~mm}$ from bregma. Scale, $1 \mathrm{~mm}$.

(C) Representative image of local virally-labeled inputs at anterior-posterior $-4.2 \mathrm{~mm}$ from bregma. Scale, $1 \mathrm{~mm}$.

(D) Representative image of a midbrain section including starter cells in the VTA as well as local inputs. Scale, $1 \mathrm{~mm}$.

(E) Starter cell distributions for each of the four experiments. The center of each oval represents the center of mass of starter cells, and the horizontal and vertical radii of the oval represent one $\mathrm{SD}$ of starter cells in the medial-lateral and dorsal-ventral axes, respectively. 
120 (F) Fraction of starter cells located in each region in the ventral midbrain. $58 \pm 6 \%$ were located in

121 the PBP, $27 \pm 6 \%$ in the PN, and $5 \pm 2 \%$ in the IF nuclei of the VTA, $8 \pm 3 \%$ in the SNc, $2 \pm 1 \%$ in the

$122 \mathrm{Rli}$, and $1 \pm 0.3 \%$ in all other regions.

$123(\mathrm{G})$ Linear regression of RABV-labeled inputs vs. number of starter cells.

$124(\mathrm{H})$ The convergence index, or ratio of inputs to starter cells, for RABV input mapping experiments

125 using $T C^{B}$ as reported in Beier et al., Cell 2015, or $T C^{66 T}$. Only long-range inputs were considered

126 to enable direct comparison between conditions. $p=0.42,95 \% \mathrm{Cl}-21.25$ to 10.24 , unpaired $t$ 127 test.

128 (I) The percentage of each local and long-distance input relative to all RABV-labeled inputs is 129 shown.

130 (J) The fraction of inputs that were long-range inputs (54 $\pm 3 \%$ ), as mapped by us in previous 131 studies, and local inputs (46 $\pm 3 \%$ ), is shown. All error bars in this figure and others throughout 132 this manuscript represent \pm 1 SEM.

133 

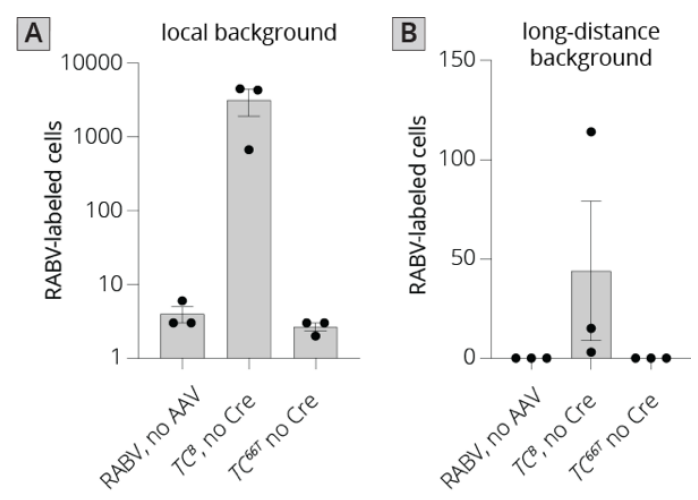

136 Supplemental Figure 1: Quantification of local and long-distance input labeling using $T C^{B}$ 137 and $T^{66 T}$.

138 (A) Local background in animals where AAVs were not injected in Cre+ mice, $T C^{B}$ was injected 139 in Cre- mice, or $T C^{66 T}$ was injected into Cre- mice.

140 (B) Long-distance background in animals where AAVs were not injected in Cre+ mice, $T C^{B}$ was 141 injected in Cre- mice, or $T C^{66 T}$ was injected into Cre- mice. 
142 AAV was injected (Supplemental Figure 1). When experiments were performed in DAT-Cre mice,

143 between 900 and 2400 starter cells were observed, and an average of 23,000 total input cells

144 were labeled per experiment (Figure 1B-C). This resulted in a signal to noise ratio of 8,710 bona

145 fide inputs : background infection. This is substantially higher than the signal : noise ratio obtained

146 using $T C^{B}$ (approximately 13: see methods for quantification.)

148 We next examined the location of starter neurons within the ventral midbrain (Figure 1D-F). The 149 majority of starter cells were located within the parabrachial pigmented nucleus (PBP: $58 \pm 6 \%$ ) 150 and paranigral nucleus (PN: $27 \pm 6 \%$ ) of the VTA. The rest of the starter neurons were located 151 within the medial aspect of the SNc (8 $\pm 3 \%$ ), interfascicular nucleus of the VTA (IF: $5 \pm 2 \%$ ), and 152 rostrolinear nucleus (Rli: $2 \pm 1 \%$ ). We then examined the ratio of inputs to starter cells, also known 153 as the convergence index, and compared it to tracing performed using $T C^{B}$. The number of RABV154 labeled inputs scaled with the number of starter cells, as expected (Figure 1G). The average 155 convergence index using $T C^{66 T}$ was approximately 8, which was not significantly different from 156 tracing performed using $T C^{B}$ (Figure $\left.1 \mathrm{H}\right)$, and also similar to the original study by Watabe-Uchida 157 and colleagues using a non-mutated TVA, where the convergence index was approximately 7 158 (Watabe-Uchida et al., 2012). However, in our study nearly half (46\%) of the inputs to VTA ${ }^{\text {DA }}$ cells 159 arose from local regions that were not quantified in our previous analysis. Though Faget and 160 colleagues excluded the VTA, SNc, SNr, red nucleus (RPC), and interpeduncular nucleus (IPN) 161 likely for precisely the reason that concerned us, these regions combined to yield $20 \%$ of the total 162 inputs to the VTA. This included about $10 \%$ of the total inputs within the VTA itself, $6 \%$ in the 163 adjacent substantia nigra, and $4 \%$ in other nearby regions.

165 However, in addition to this $20 \%$ of inputs in regions immediately adjacent to the injection site, 166 given that AAVs can exhibit substantial spread from the injection site we were concerned that 167 non-Cre-dependent, EnvA-mediated infection extended beyond these regions to several other 168 midbrain regions. To assess if this was indeed a problem, we compared our data to that published 169 by Faget and colleagues for three separate groups of regions: 1) Those excluded in the previous 170 study, 2) Midbrain/hindbrain regions excluded by us previously but not by the previous study, 3) 171 long-range inputs included by both studies. Using dimensional reduction techniques to compare 172 the overall labeling patterns within these three groups, we found that our data mixed with that 173 from the Faget et al. study for long-range inputs (comparison 3) suggesting that the data were 174 comparable. However, data from the two studies segregated for both the excluded (comparison 175 1) and questionable (comparison 2) brain regions (Supplemental Figure 2). These results support 
176 the likelihood that our method of using a modified, less efficient version of TVA is necessary to

177 properly examine the local input landscape in the ventral midbrain.

\section{Local inputs to VTA ${ }^{\mathrm{DA}}$ cells have differential associations with long-range inputs}

180 Given the large fraction of total inputs to VTA ${ }^{\mathrm{DA}}$ that are local, we wanted to explore the nature of 181 these connections and by extension, how they may influence VTA ${ }^{\mathrm{DA}}$ cells. Long-range inputs to

182 DA neurons measured using RABV tracing have been published several times (Beier et al., 2015;

183 Faget et al., 2016; Watabe-Uchida et al., 2012). More recently, DA neurons have been subdivided 184 by output site, and the inputs to particular subpopulations compared to one another (Beier et al., 185 2015, 2019; Derdeyn et al., 2022; Lammel et al., 2012; Menegas et al., 2015).These analyses 186 have enabled us to understand how input patterns relate to one another, and which inputs are 187 biased onto which sets of VTA ${ }^{\mathrm{DA}}$ cells. We can leverage these previous analyses to explore with 188 which long-range inputs each set of local inputs associates, and by extension, which sets of DA cells may be predominately targeted by each set of inputs.

We first used Uniform Manifold Approximation and Projection (UMAP) to identify the clustering relationships of different input sites. We found that local input sites intermingled with long-distance inputs (Figure 2A). This is expected, and suggests that local inputs co-organize with long-distance

194 inputs, rather than being a separate set of circuits. Given that we used only 4 brains for these 195 UMAP analyses, which is a relatively small number for accurately capturing variance in the dataset, we wanted to test how robust our UMAP embedding was in capturing the relationships between brain regions. We recently performed a similar analysis on a 76 brain dataset that included long-distance RABV-labeled inputs to VTA cells based on projection, neurochemical phenotype, or a combination of these factors (Derdeyn et al., 2022). Since UMAP embeddings can be somewhat stochastic due to their reliance on initial seeding conditions, we also computed the distance between points relative to the maximum distance between any two points in each embedding, over 20 embeddings, then averaged across all embeddings for our four brain dataset, as done previously for the 76 brain dataset (Derdeyn et al., 2022). We found that the region associations were robust, with only three long-range input regions - the nucleus accumbens medial shell (NAcMed), nucleus accumbens core (NAcCore), and extended amygdala (EAM) associating with different groups of inputs across conditions (Figure $2 \mathrm{~B}-\mathrm{C}$ ). While the results were not identical, these data suggest that our four brain dataset could be compared with reasonable 208 confidence to our previous dataset. 

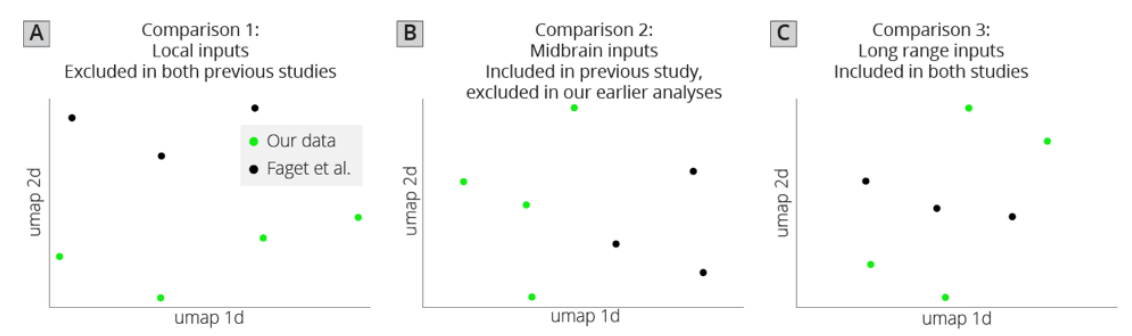

\section{Supplemental Figure 2: UMAP analysis of VTA ${ }^{\mathrm{DA}}$ input tracing datasets.}

212 (A) UMAP embedding of brains using only inputs excluded in our previous studies as well as in 213 Faget et al. These included the VTA, substantia nigra (SNc/SNr), IPN, RPC, and MHb.

214 (B) UMAP embedding of local inputs included in Faget et al., but excluded from our previous 215 studies. These inputs included the PSth, MM, SuM, MnR, PPTg, RMg, RRF, PT, InC, PAG, RI, 216 VTg, colliculus (IC + SC), MiTg, SPTg, ATg, DLL, PnC, PnO, RtTg, and SubB.

217 (C) UMAP embedding of long-range inputs from our study and Faget et al. Regions of the 218 isocortex anterior to the corpus collosum were combined to make the anterior cortex. The EAM 219 and DCN were excluded from the analysis as these were not contained in both datasets, and the $220 \mathrm{MHb}$ was excluded from this dataset because it was contained in the excluded dataset (panel A). 

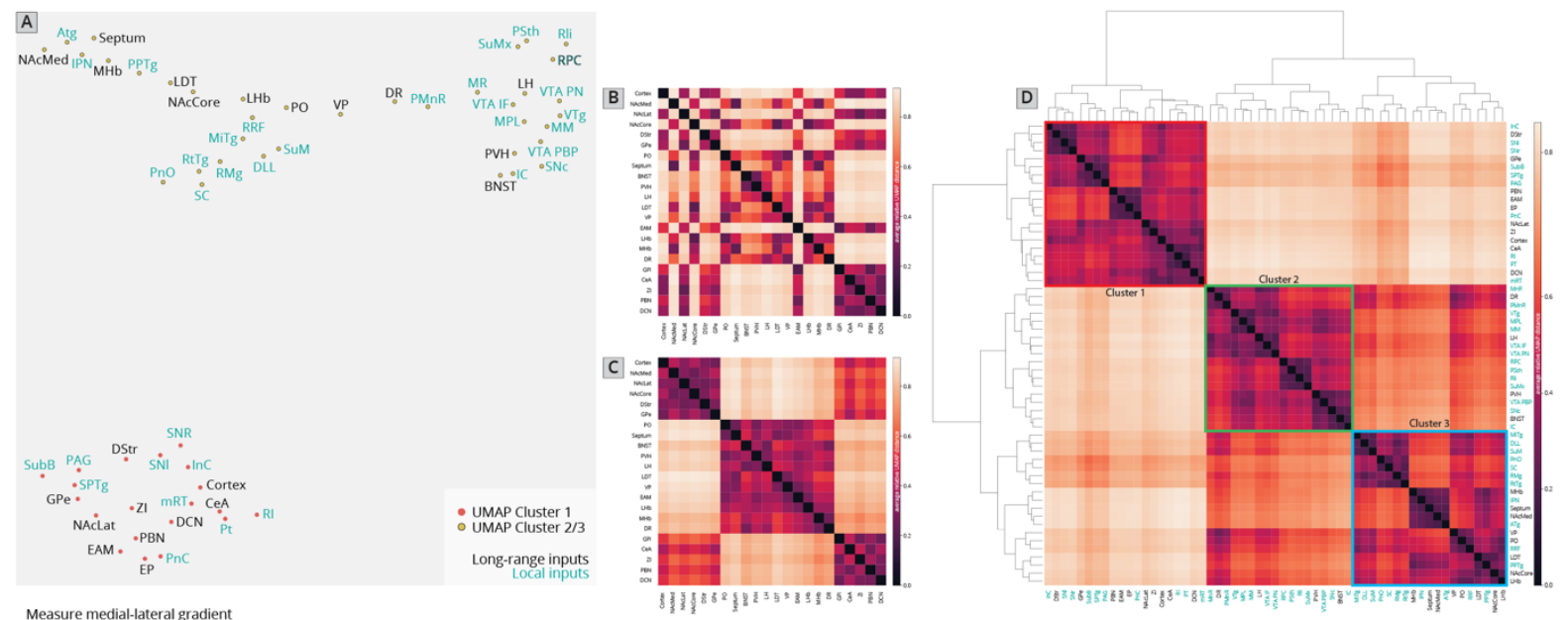

Measure medial-lateral gradient
of each projection in the VIA
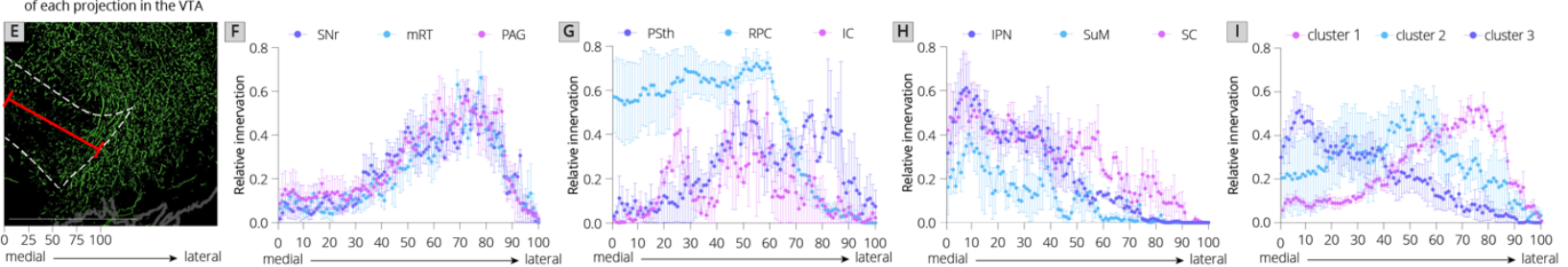

Figure 2: Association of local inputs to VTA ${ }^{\mathrm{DA}}$ cells with long-range inputs.

224 (A) Input regions are plotted in UMAP space, embedded with respect to z-scored counts across

225 mouse brains. Clusters represent inputs with similar patterns of variation across the cohort.

226 (B) Heatmap of pairwise distances (averaged across 20 UMAP embeddings) for the RABV input 227 data, including only long-distance inputs to enable comparison to our 76-brain dataset, where 228 only long-distance inputs were analyzed.

229 (C) Heatmap of pairwise distances from an aggregated UMAP analysis of our 76 brain dataset,

230 from Derdeyn et al. 2022, for purposes of comparison.

231 (D) Heatmap of pairwise distances for the RABV input data, including both local and long-distance

232 inputs. Regions are grouped according to hierarchical clusters. Clusters are highlighted to match 233 the clusters in the UMAP plot.

234 (E) Schematic of how relative innervation of the medial-lateral axis of the VTA was quantified.

235 Scale, $1 \mathrm{~mm}$. Images used for quantification of the data in panels E-I were obtained from the Allen

236 Mouse Brain Connectivity Atlas.

237 (F) Relative innervation across the medial-lateral axis for UMAP cluster 1.

238 (G) Relative innervation across the medial-lateral axis for UMAP cluster 2.

$239(\mathrm{H})$ Relative innervation across the medial-lateral axis for UMAP cluster 3.

240 (I) Relative innervation across the medial-lateral axis for each UMAP cluster, representing the 241 average of the three individual quantified input regions shown in $\mathrm{F}-\mathrm{H}$. 
242 Similar to our 76 brain dataset, we observed three clusters of input regions (Figure 2D). The first

243 is the set of brain regions that predominately project lateral or dorsal to the VTA (Derdeyn et al.,

244 2022). The regions that project laterally include inputs from the basal ganglia such as the DStr,

245 NAcLat, GPe, and cortex. Those that project dorsally include the entopeduncular nucleus (EP),

246 zona incerta ( $\mathrm{ZI}$ ), and deep cerebellar nuclei (DCN). The local inputs that associate with this

247 cluster include mostly those located dorsally and laterally to the VTA, including the periaqueductal

248 gray (PAG), midbrain reticular nucleus (mRT), interstitial nucleus of Cajal (InC), subpeduncular

249 tegmental nucleus (SPTg), subbrachial nucleus (SubB), SNr, and SNI. To test if the local inputs

250 we identified indeed projected laterally to the VTA, we mapped out the relative innervation by the

251 SNr, mRT, and PAG of the VTA across the medial-lateral gradient, as done previously for long-

252 range inputs, using the Allen Mouse Brain Connectivity Atlas (Figure 2E) (Beier et al., 2019). We

253 indeed observed that three selected inputs in cluster 1 - the SNr, mRT, and PAG - displayed a

254 lateral bias in the VTA (Figure 2F, Supplemental Figure 3).

256 The second cluster of inputs includes mostly regions that project uniformly across the VTA, 257 including the lateral hypothalamus ( $\mathrm{LH})$, paraventricular hypothalamus $(\mathrm{PVH})$, and bed nucleus 258 of the stria terminalis (BNST) (Derdeyn et al., 2022). The local inputs associating with this cluster 259 include inputs from all of the subregions of the VTA itself (PBP, PN, IF), and other inputs located 260 along the midline [median raphe (MnR), supramammillary decussation (SuMx), medial 261 mammillary nucleus (MM)] as well as those located just laterally to the midline such as the red 262 nucleus (RPC), ventral tegmental nucleus (VTg), inferior colliculus (IC), and parasubthalamic 263 nucleus (PSth). While more varied in their individual projections, the PSth, RPC, and IC show a 264 more consistent projection across the medial-lateral axis of the VTA (Figure 2G).

266 The final cluster of inputs mostly includes those that project medially within the VTA. This includes 267 the $\mathrm{MHb}$ and lateral habenula ( $\mathrm{LHb}$ ), septum, preoptic nucleus (PO), and laterodorsal tegmentum 268 (LDT). Local inputs associating with this group include midline structures such as the IPN, raphe 269 magnus (RMg), supramammillary nucleus (SuM), structures near the midline such as the 270 reticulotegmental nucleus (RtTg), anterior tegmental nucleus (ATg), superior colliculus (SC), 271 slightly more lateral structures such as the retrorubal field (RRF) and pedunculopontine tegmental 272 nucleus (PPTg), or inputs with lower counts such as the microcellular tegmental nucleus (MiTg) 273 and the dorsal nucleus of the lateral lemniscus (DLL). The IPN, SuM, and SC all showed a medial 274 bias in the VTA (Figure $2 \mathrm{H}$ ). The difference in medial-lateral preference among the three clusters 275 was especially clear when averaged across the three tested regions and plotted together (Figure 

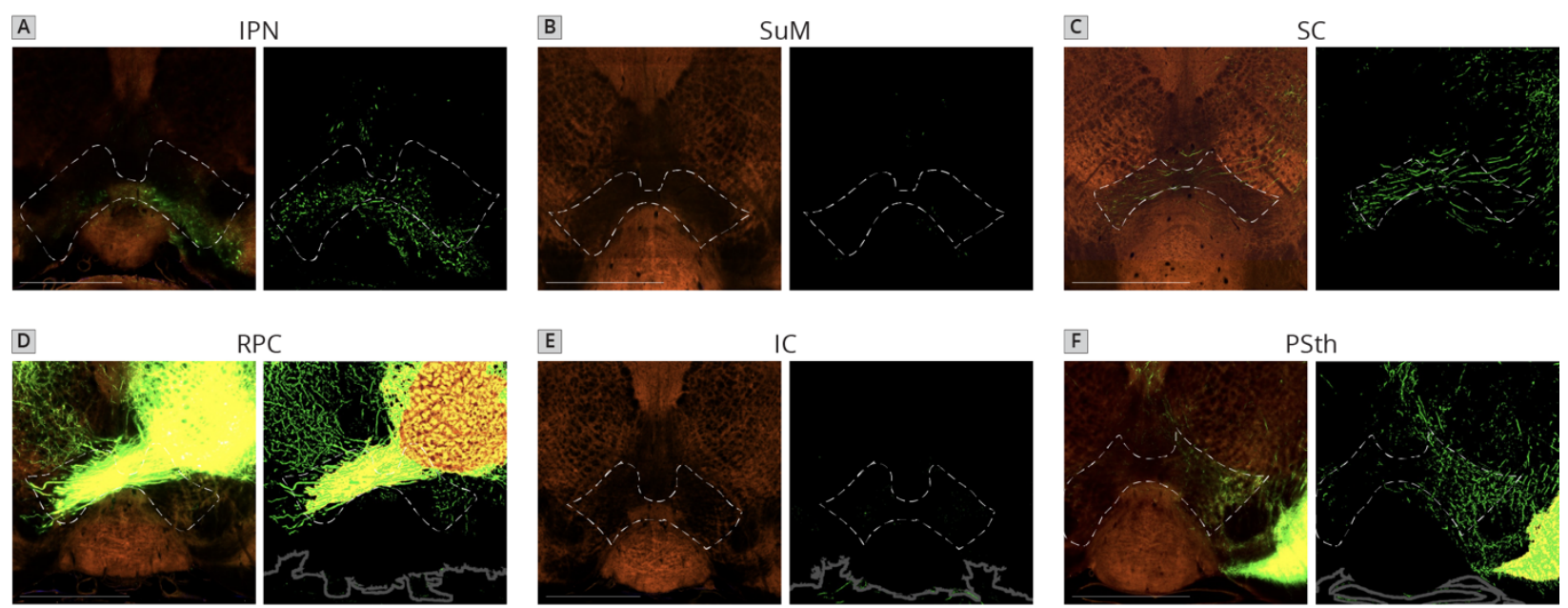

RPC
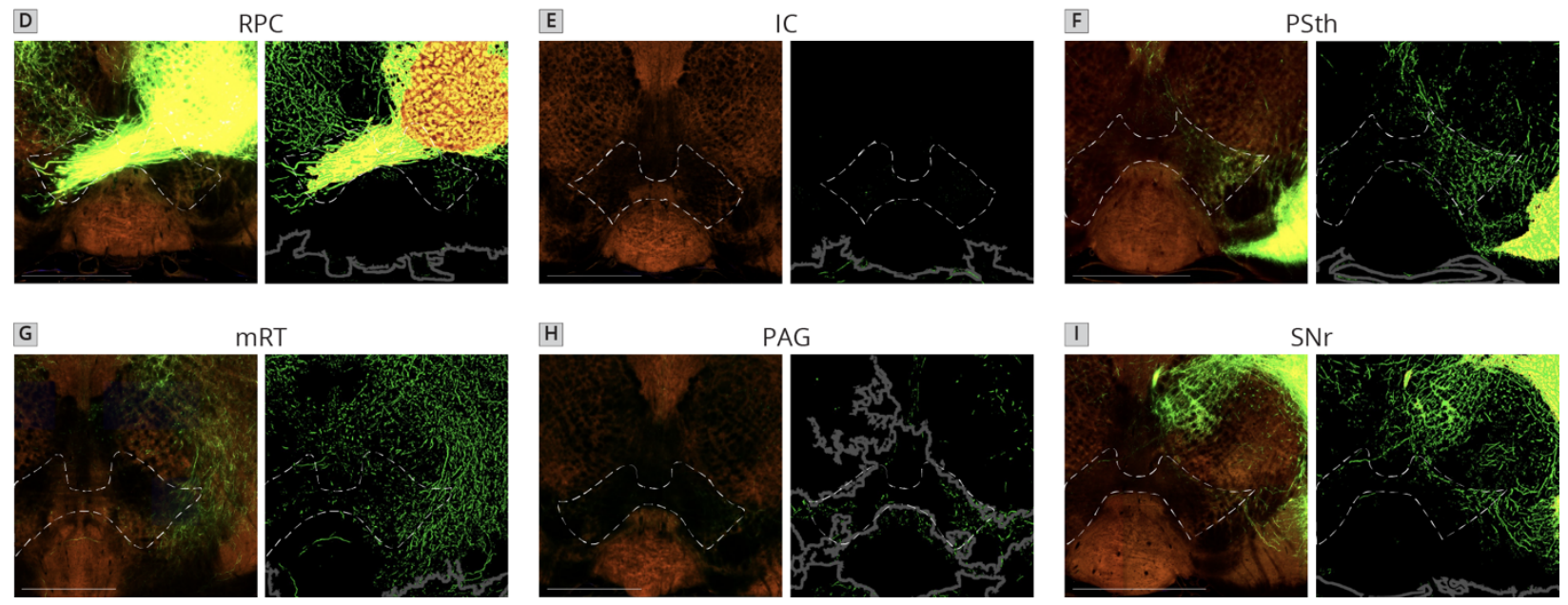

277 Supplemental Figure 3: Sample images of projections in the VTA from each of the 9

278 quantified local inputs to VTA ${ }^{\text {DA }}$ cells. Images are from axons of labeled cells in the (A) IPN,

279 (B) SuM, (C) SC, (D) RPC, (E) IC, (F) PSth, (G) mRT, (H) PAG, (I) SNr. 
281 2I). These data in sum suggest a topographical organization of inputs to the VTA that applies to 282 both global and local inputs, whereby inputs segregate based on their projection medially or 283 laterally within the VTA, as we have found previously with long-range inputs (Beier et al., 2015, 284 2019; Derdeyn et al., 2022). Here, we extend these observations by including all inputs to the 285 VTA, regardless of distance from injection site.

\section{Local and distributed GABAergic inhibition to VTA ${ }^{\mathrm{DA}}$ cells}

288 We next wanted to assess the location of local GABAergic inhibition to the VTA. GABA neurons 289 functionally oppose DA neurons, providing a punishment and aversion signal (Bouarab et al., 290 2019; Tan et al., 2012). The majority of studies of inhibition to VTA ${ }^{\text {DA }}$ cells have focused on either 291 GABA neurons within the VTA or on an anatomically poorly-defined structure referred to as the 292 rostromedial tegmental nucleus (RMTg) (Jhou 2005; Jhou et al., 2009a; Jhou et al., 2009b; 293 Kaufling et al., 2009). While extensive efforts have mapped the inputs, including inhibitory cell 294 inputs to the VTA (Geisler et al., 2007; Geisler \& Zahm, 2005; Phillipson, 1979; Sesack \& Grace, 295 2010; Swanson, 2000; Zahm et al., 2011), these methods lacked the connectivity information 296 afforded by RABV. We therefore performed our local mapping experiments in combination with fluorescent in situ hybridization against the GABAergic markers GAD1 and GAD2 in order to provide a quantitative map of inhibition onto VTA ${ }^{\mathrm{DA}}$ cells (Figure $3 \mathrm{~A}-\mathrm{B}$ ).

In order to assess how this local input map would relate to our whole-brain local/global maps elucidated in Figure 1, we first compared the location of starter neurons in the VTA between experiments (Figure 3C-D). The majority of starter neurons were located within the PBP nucleus of the VTA $(63 \pm 1 \%)$, followed by VTA PN $(15 \pm 1 \%)$, SNc $(11 \pm 2 \%)$, VTA IF $(6 \pm 1 \%)$, and Rli (3 $\pm 1 \%$ ). This was a similar distribution to our whole-brain mapping experiments (Figure $1 \mathrm{~F}$ ), enabling direct comparison of these datasets.

We counted all RABV-labeled neurons in regions local to the VTA, and assessed their co-staining with GAD1/GAD2 markers as well as $T C^{66 T}$. GABAergic input neurons were identified as those that were negative for $T C^{66 T}$ and positive for GAD1/GAD2 (Figure 3E). We found that, surprisingly,

310 the SNr is the single largest source of inhibition onto the cells that we targeted, representing 20.1 $311 \pm 1.4 \%$ of all inhibitory inputs (Figure 3F-G). Given that the VTA contains $84 \%$ of starter cells and 312 SNc contained only $11 \%$ of starter cells, and most of these SNc starter cells were on the border 313 of the lateral VTA/medial SNc, these SNr neurons almost certainly inhibit both SNc and VTA 

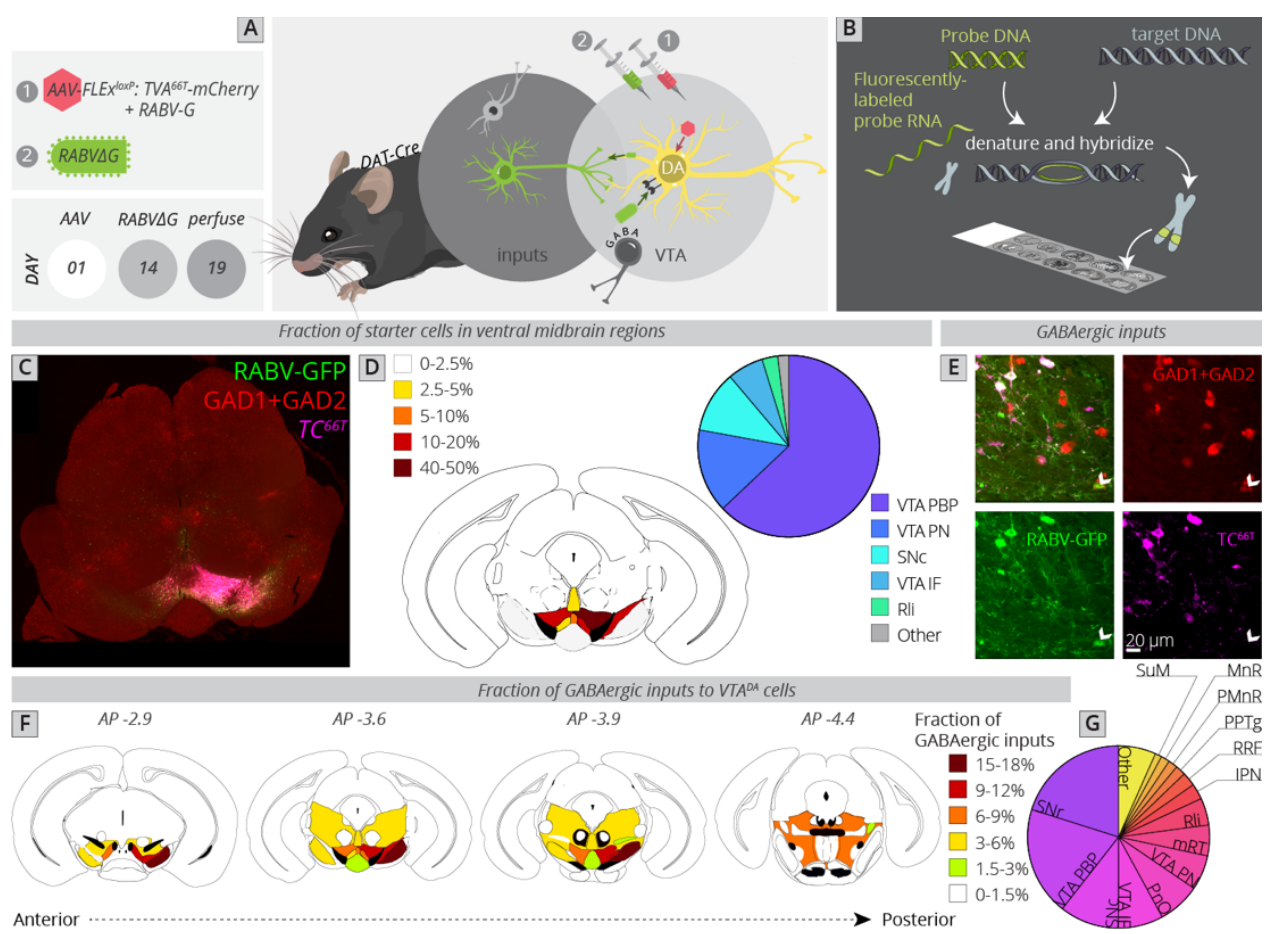

316 Figure 3: Local and distributed GABAergic inhibition to VTA ${ }^{\mathrm{DA}}$ cells.

317 (A) Strategy for viral mapping combined with fluorescent in-situ hybridization with probes for 318 GAD1 and GAD2.

319 (B) Sample histology section of the midbrain near the injection site. Green = RABV, red $=\mathrm{FISH}$ 320 for GAD1/GAD2, magenta $=T C^{66 T}$. Scale, $1 \mathrm{~mm}$.

321 (C) Breakdown of starter cell location in different midbrain nuclei. $63 \pm 1 \%$ were located within

322 the PBP nucleus of the VTA, $15 \pm 1 \%$ in the VTA PN, $11 \pm 2 \%$ in the SNc, $6 \pm 1 \%$ in the VTA IF,

$3233 \pm 1 \%$ in the Rli, and $2 \pm 0.3 \%$ in all other regions.

324 (D) Example histology image of labeled cells, with the arrowhead pointing to a RABV+, 325 GAD1/GAD2+, TC ${ }^{66 T}$ - cell.

326 (E) Schematic heatmap of GABAergic input location throughout the ventral midbrain. Colors 327 correspond to the total \% of local GABAergic inputs located in that particular region. Data from 328 each hemisphere is reported separately. The injection occurred in the right hemisphere.

329 (F) Pie chart representing the total fraction of GABAergic inputs located in each region. Data from 330 both hemispheres are combined. $20.1 \pm 1.4 \%$ of all inhibitory inputs are from the SNr, $19.3 \pm 0.7 \%$ 331 from the VTA PBP, SNc $10.5 \pm 2.4 \%$, VTA IF $8.0 \pm 2.6$, VTA PN $6.2 \pm 0.6$, PnO $7.5 \pm 2.4 \%$, mRT $3325.5 \pm 1.3 \%$, Rli $4.8 \pm 0.5$, IPN $2.8 \pm 1.8$, RRF $2.5 \pm 1.1 \%$, PPTg $1.8 \pm 0.8 \%$, PMnR $1.6 \pm 0.3 \%$, MnR $3331.2 \pm 1.2 \%$, SuM $1.2 \pm 0.2$, and $6.6 \pm 0.8$ in all other regions. 
neurons. The majority of labeled SNr cells were located in the ventral-medial aspect of the SNr, near to the VTA. The second largest source of input was the PBP nucleus of the VTA (19.3 \pm $0.7 \%)$, followed by the SNc $(10.5 \pm 2.4 \%)$. These three inputs represented $50 \%$ of the total inhibition of VTA ${ }^{\mathrm{DA}}$ neurons. With the addition of the IF $(8.0 \pm 2.6)$ and PN $(6.2 \pm 0.6)$ nuclei of the VTA, $64 \%$ of inhibitory neurons are from regions within or immediately adjacent to the VTA.

In addition to inhibitory input from the above regions, VTA ${ }^{\mathrm{DA}}$ neurons received inhibitory inputs from distributed sites across numerous regions posterior to the VTA. These included the pontine reticular nucleus, oral part (PnO: $7.5 \pm 2.4 \%), \operatorname{mRT}(5.5 \pm 1.3 \%), \operatorname{Rli}(4.8 \pm 0.5)$, IPN $(2.8 \pm 1.8), \operatorname{RRF}$ (2.5 $\pm 1.1 \%)$, PPTg (1.8 $\pm 0.8 \%)$, paramedian raphe nucleus (PMnR: $1.6 \pm 0.3 \%), \operatorname{MnR}(1.2 \pm 1.2 \%)$, and SuM (1.2 \pm 0.2$)$. Several of these regions are thought to contribute to the RMTg, consistent with their known behavioral role in modulating VTA ${ }^{\mathrm{DA}}$ cells. However, it is notable that at least quantitatively, they represent a minor fraction of total inhibitory inputs to VTA ${ }^{\mathrm{DA}}$ cells.

\section{Midbrain DA neurons exhibit extensive interconnectivity}

$350 V_{T A}{ }^{\text {GABA }}$ neurons in total comprised about $10 \%$ of the total inputs to VTA ${ }^{\text {DA }}$ cells (Figure 1), and about $33 \%$ of the total local GABAergic input to the VTA ${ }^{\text {DA }}$ cells (Figure 3 ). However, in addition to the VTA ${ }^{G A B A}$ input, there is evidence that $V T A^{D A}$ cells also receive inputs from local glutamatergic (Dobi et al., 2010) as well as DA cells. Physiological evidence has shown that DA neurons release DA within the midbrain via somatodendritic release (Bayer \& Pickel, 1990; Groves \& Linder, 1983). Locally released DA then binds D2 autoreceptors expressed on DA cells, suppressing their activity. We wanted to test if DA neurons were connected by conventional means, and if so, what the topology of DA-DA neuron connectivity is in the ventral midbrain.

The challenge of assessing DA-DA connectivity is to distinguish cells that express TVA and thus could serve as starter neurons, and those that did not (and thus represent bona-fide inputs). We previously published the extent of spread of both $A A V_{5}-C A G-F L E x^{\operatorname{lox} P}-T C^{B}$ and $A A V_{8}-C A G-$ $F L E x^{\text {loxP }}-R A B V-G$ in the ventral midbrain given the exact injection coordinates used in this study, which provided a quantified radius of spread for each virus (Beier et al., 2015, 2019). We stained brain sections with both an anti-tyrosine hydroxylase $(\mathrm{TH})$ antibody to label DA cells, as well as an mCherry antibody to delineate $T C^{66 T}$-expressing neurons (Figure 4A-E). We then assessed regions outside of the sphere of TVA spread that were either anterior in the VTA, posterior/medial or posterior/lateral in the VTA, as well as in the nearly RRF, and tested for how many neurons costained with $\mathrm{TH}$. We found that about $40 \%$ of inputs in each region that did not express clear 

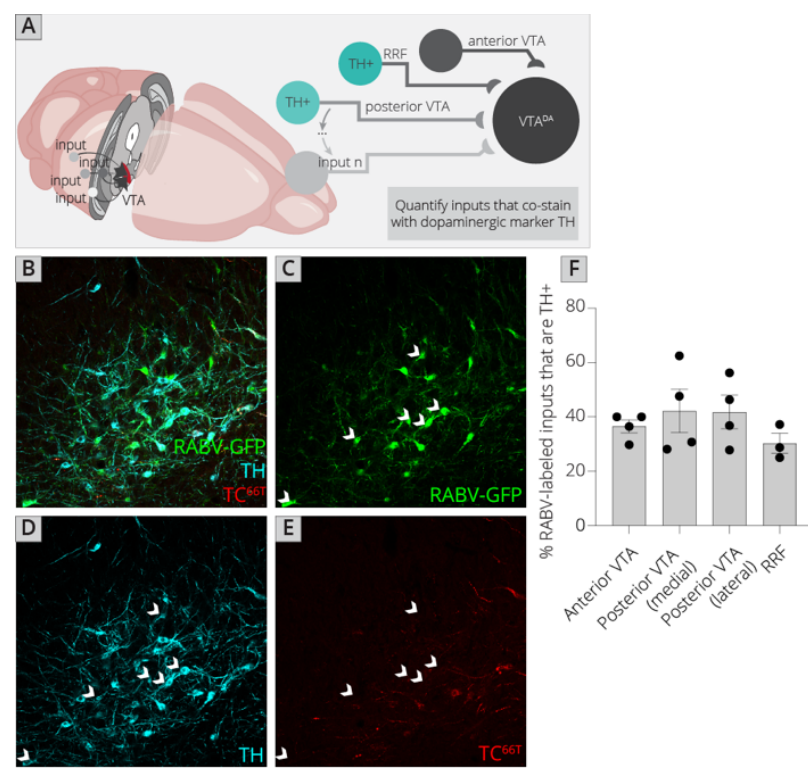

370 Figure 4: Connections between DA cells in the ventral midbrain.

371 (A) Schematic of experiments for assessing potential DA-DA connections. Brain sections were

372 stained for TH and mCherry and local inputs in the A8 (RRF) and A10 (VTA) regions were

373 assessed for co-labeling with TH and mCherry. Cells that were GFP+, $\mathrm{TH}+$, and mCherry-, were

374 considered DAergic inputs.

375 (B-E) Example histology image of labeled cells in the RRF. Green $=$ GFP, cyan $=\mathrm{TH}$, red $=$ 376 mCherry. Scale, $50 \mu \mathrm{m}$.

377 (F) Quantification of $\mathrm{TH}+$ inputs in four different midbrain regions. 
380 mCherry protein, even after antibody amplification, co-stained with TH (Figure 4F). That this 381 number did not substantially differ regardless of whether the site was nearer to the injection site

382 (anterior, posterior/medial) or further away (posterior/lateral, RRF) provides further evidence that 383 RABV labeling was not due to direct TVA-mediated uptake, as in that case we would expect a 384 higher percentage of $\mathrm{TH}+$ cells in regions near the injection site. Our results suggest that in 385 addition to a substantial GABAergic input from the VTA to local VTA ${ }^{\text {DA }}$ cells, VTA ${ }^{\text {DA }}$ neurons 386 receive a large input from other DA cells that comprises approximately $4-5 \%$ of the total inputs to 387 VTA ${ }^{\text {DA }}$ cells $(11 \%$ of total inputs from the VTA/RRF $\times 40 \%$ that are $\mathrm{TH}+$ ). These results suggest 388 that there is indeed a substantial level of connectivity between DA neurons throughout the VTA 389 and adjacent structures.

\section{Sources of serotonergic inputs to VTA ${ }^{\mathrm{DA}}$ cells}

392 The brain's neuromodulatory systems, including cells that release the monoamines DA, serotonin, 393 or norepinephrine, are extensively interconnected (Hensler et al., 2013). In particular, serotonin 394 (5-HT) plays a key role in the modulation of the DA system. A number of studies using classical methods such as small molecule and dye tracers have found that the VTA receives strong innervation from cells in the B7 region, also known as the dorsal raphe (DR) (Phillipson, 1979). However, the methods employed by these studies do not distinguish connected cells from passing fibers, or inputs connected to non-DA cells. Several studies including our own have demonstrated that serotonergic neurons in the DR do directly connect to both DA and GABA cells in the VTA (Beier et al., 2015; Liu et al., 2014; McDevitt et al., 2014; Qi et al., 2014; Wang et al., 2019). However, serotonergic input likely arises from other serotonergic nuclei besides the DR. Where the serotonergic neuron input is arising from, what the density of this innervation is, and the fraction of serotonergic innervation provided by each region is not known. Since several of the serotonergic cell-containing brain regions, including the pontine tegmentum (B9) and MnR (B8) regions are located nearby the VTA ${ }^{\mathrm{DA}}$ cells, connectivity could not be discerned using viral mapping studies that employ the standard FLEx ${ }^{\text {loxP }} / D I O$ TVA to mark starter cells. Therefore, our approach is uniquely suited to address this question.

We mapped inputs to VTA ${ }^{\mathrm{DA}}$ neurons, as before, and co-stained neurons in the midbrain and 410 hindbrain with an antibody to tryptophan hydroxylase 2 (Tph2), which marks serotonergic neurons 411 (Figure 5A-B). We examined both hemispheres independently, except for the RMg (B3), DR (B7), 412 and $\mathrm{MnR}$ (B8), as these cell populations are located along the midline. We observed that 413 approximately $30 \%$ of cells in each region co-stained with Tph2, with a high of $37 \%$ and a low of 


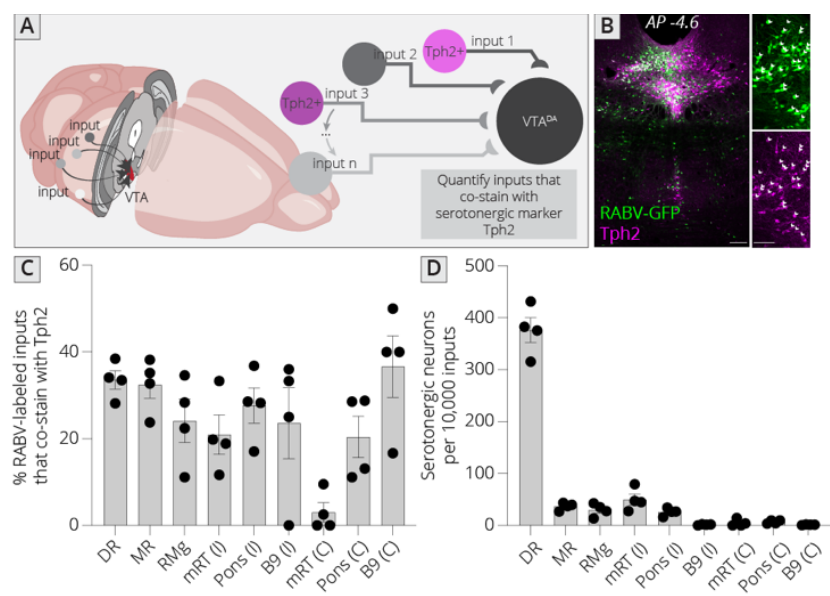

415 Figure 5: Identifying location of serotonergic inputs to VTA ${ }^{\mathrm{DA}}$ cells.

416 (A) Schematic of experiments for assessing potential serotonergic inputs. Brain sections were

417 stained for Tph2, and inputs in the B3, B5, B6, B8, and B9 regions were assessed for co-labeling

418 with Tph2. Cells that were GFP+ and Tph2+ were considered serotonergic inputs.

419 (B) Example histology image of labeled cells in the midbrain. Green = GFP, magenta = Tph2.

420 Scale, $50 \mu \mathrm{m}$.

421 (C) Percentage of RABV-labeled inputs in each region that co-stained with Tph2.

422 (D) The number of serotonergic inputs in each quantified region, normalized to 10,000 RABV-

423 labeled inputs. 
$42620 \%$ (Figure 5C). This number did not vary substantially, regardless of the region or hemisphere,

427 suggesting that the fraction of cells providing direct connections onto VTA ${ }^{\text {DA }}$ neurons that are

428 serotonergic within each region is relatively constant across all serotonergic nuclei. We also want

429 to assess the fraction of total input provided by each set of serotonergic cells. We therefore

430 normalized the number of serotonergic inputs in each region per 10,000 RABV-labeled input cells.

431 This analysis showed that the vast majority of serotonergic inputs to the VTA indeed arise from

432 the DR (376 \pm 24 per 10,000 inputs; Figure 5D). The regions providing the next largest quantitative

433 serotonergic inputs are the $\mathrm{mRT}(\mathrm{B6} ; 50 \pm 11$ inputs per 10,000), MnR (37 \pm 4 inputs per 10,000),

$434 \operatorname{RMg}(30 \pm 6$ inputs per 10,000), and pons (B5; $26 \pm 4$ inputs per 10,000). Therefore, while the

435 majority of serotonergic inputs do arise from the DR, VTA ${ }^{\text {AA }}$ cells also receive distributed

436 serotonergic input from several other midbrain and hindbrain nuclei that together represent

437 approximately $1.5 \%$ of total inputs to VTA ${ }^{\text {DA }}$ cells.

\section{DISCUSSION}

440 In this study, we provide a comprehensive local and global input map to VTA ${ }^{\text {DA }}$ neurons. We

441 focused on local inputs to the VTA that were inaccessible using previous approaches, and

442 quantified where GABAergic, DAergic, and serotonergic inputs arose in the midbrain and

443 hindbrain. Our study provides the first comprehensive map of these connections to VTA ${ }^{\text {DA }}$

444 neurons and thus provides a valuable resource for future studies of the VTA as well as a template

445 for mapping both local and global inputs to other structures in the brain.

447 Inputs to DA cells in the VTA have been previously mapped (Beier et al., 2015; Faget et al., 2016;

448 Watabe-Uchida et al., 2012). However, each of these studies reported a limited number of input 449 regions, as the issue of local background was likely recognized in each study. The report from

450 Watabe-Uchida and colleagues included a much reduced local input labeling relative to this study,

451 perhaps due to the limited reporting of input regions. Most importantly, nearly every study to date,

452 including the three cited above, used a wild-type version of the TVA protein that would likely yield

453 hundreds to thousands of non-Cre-dependent, TVA-facilitated infections in the midbrain near the

454 injection site. Our approach using $T C^{66 T}$ to reduce non-Cre-mediated infections near the site of

455 injection enabled high-resolution, low-noise analysis of connectivity to VTA ${ }^{\mathrm{DA}}$ cells. The fact that

456 nearly $50 \%$ of inputs are from local brain sites highlights the importance of local connectivity within

457 the midbrain. That many of the brain regions are much less studied than their forebrain

458 counterparts represents an opportunity to further study inputs from the midbrain and hindbrain 
that provide quantitatively substantial inputs to VTA ${ }^{\mathrm{DA}}$ neurons yet whose functions remain largely unknown.

\section{Relationships with different long-range input sites}

463 We previously mapped inputs to VTA neurons based on output site and neurochemical phenotype, 464 amassing a 76 brain dataset that enabled us to dissect the sources that influence patterns of 465 inputs to different cells in the VTA (Beier et al., 2015, 2019; Derdeyn et al., 2022). In this study, 466 we wanted to map the local inputs onto these scaffolds to observe if local inputs have biases 467 towards specific VTA ${ }^{\mathrm{DA}}$ subpopulations. We demonstrated here using a subset of local input 468 regions that the three clusters of input regions arising from our UMAP analysis were related to 469 spatial projection to the VTA. We used output tracing experiments from three representative local 470 input regions from the Allen Mouse Brain Connectivity Atlas, as the number we could select was 471 quite limited. This was due to the fact that most of the local input brain regions are small, and thus 472 without defined Cre cell populations to target each population, more often than not the desired 473 brain region represented only a small fraction of the total cells expressing GFP. Even so, the brain 474 regions that we identified that did contain at least 2 injections relatively specifically targeting the 475 desired regions reinforced our interpretation that the UMAP clusters represent different medial476 lateral projection patterns in the VTA.

We had previously linked biases in long-range inputs to different VTA cell populations, and showed that these biases were related to stereotyped projection archetypes in the VTA (Derdeyn et al., 2022). Namely, inputs that project laterally to the VTA predominantly innervate VTA $^{\mathrm{DA}} \rightarrow$ NAcLat cells, those that uniformly innervate the VTA are biased onto VTA ${ }^{\mathrm{DA}} \rightarrow$ Amygdala cells, those projecting ventromedially preferentially target VTA $\rightarrow$ NAcMed cells, and those that project ventromedially target VTA ${ }^{\mathrm{DA}} \rightarrow$ medial prefrontal cortex (mPFC) cells (Derdeyn et al., 2022). Though the four brains used for this analysis were many fewer than the 76 brain dataset used previously, we were able to recapitulate the main associations between brain regions, supporting the validity of our interpretations. This thus suggests that local inputs to cluster 1 (SNr, SNI, mRT, 487 SPTg, SubB, PnC, RI, PT, InC) principally target VTA ${ }^{\mathrm{DA}} \rightarrow$ NAcLat cells, those in cluster 2 (PSth, 488 IC, RPC, VTA PBP, PN, IF, MnR, Rli, SNc, PMnR, VTg, MPL, MM, SuMx) predominately 489 innervate $\mathrm{VTA}^{\mathrm{DA}} \rightarrow$ Amygdala neurons, and those in cluster 3 (IPN, SuM, SC, PnO, ATg, RMg, 490 PPTg, RRF, RtTg, MiTg, DLL) are biased onto VTA ${ }^{\mathrm{DA}} \rightarrow \mathrm{NAcMed}$ or VTA ${ }^{\mathrm{DA}} \rightarrow \mathrm{mPFC}$ cells. One 491 important limitation is that our analyses here are limited to the medial-lateral axis in the VTA. We 492 previously showed that projections demonstrate both a medial-lateral and dorsal-ventral gradient 
493 in the VTA (Derdeyn et al., 2022). We chose to focus here on the medial-lateral axis as that is the

494 principal axis of variation for long-range inputs in the VTA (Derdeyn et al., 2022). Our analyses

495 have generally not had sufficient anterior-posterior resolution since the location of starter cells,

496 which was critical to the original observation of spatial gradients in inputs to the VTA, was obtained

497 using serial $60 \mu \mathrm{m}$ coronal sections in the VTA (Beier et al., 2015, 2019). Therefore, the local

498 inputs mapped here may have additional gradients along the dorsal-ventral or anterior-posterior

499 axes that were not captured in our analysis.

DA-DA cell interconnectivity

502 In addition to local GABAergic inputs, we also found a large number of DA-DA cell connections in 503 the VTA. DA neurons are known to signal to one another through release of DA, which is thought 504 to signal through volume transmission (Groves \& Linder, 1983; Hajdu et al., 1973; Wilson et al., 505 1977). It is therefore of interest to consider, given that the dominant hypothesis is that RABV is transmitted via synapses, how this DA-DA neuron transmission may occur. In a study mapping connections to direct and indirect pathway neurons in the dorsal striatum, RABV was also observed to transmit to DA cells (Wall et al., 2013). However, this mode of transmission occurs approximately 10-fold less efficiently than when RABV was injected directly into the dorsal striatum. However, it is not certain that inter-midbrain DA transmission occurs via classic volume transmission and not focal release/signaling of DA. For example, DA levels appear to rise to $\geq 10$ $\mathrm{uM}$ for brief periods of $\leq 100 \mathrm{~ms}$ (Ford et al., 2009). This rapid on/off kinetics of DA signaling is inconsistent with DA signaling at a distance (Beckstead et al., 2004). Furthermore, spontaneous exocytotic, GPCR-mediated signaling was observed in SNc ${ }^{\mathrm{DA}}$ neurons (Gantz et al., 2013). This evidence indicates that, like traditional ligand-gated transmission, GPCR-mediated transmission can occur in a point to point fashion, similar to a classic synaptic mechanism. More recent evidence using the DA sensor dLight and a photoactivatable D2 receptor ligand also supports the hypothesis that DA release occurs from highly specific sites (Condon et al., 2021). From our experiments we cannot infer the nature of DA release or contacts between DA cells. However, our results also support the possibility of specific contacts between DA cells through which RABV transmission can occur. We estimate that approximately $4-5 \%$ of total RABV-labeled inputs are

522 from DA cells. If this number is also influenced by a ten-fold reduction in efficiency of transmission, 523 as estimated from Wall and colleagues, this would indicate that the presence of DA terminals, 524 and thus the potential for DA influence of other VTA ${ }^{\mathrm{DA}}$ cells is extraordinarily high. 
526 This interconnectivity of DA cells throughout the ventral midbrain also has implications for tract

527 tracing studies. Several studies, including the earliest input-output viral-genetic mapping study of

528 the VTA defined output cells by injection of RABV into a projection site, and enabled spread of

529 RABV from VTA cells via injection of an AAV expressing RABV-G into the ventral midbrain of

530 DAT-Cre mice (Lammel et al., 2012). According to this strategy, any DA cell expressing RABV-G

531 could serve as a starter cell. Thus, for example when RABV was injected into NAcLat, if $532 \mathrm{VTA}^{\mathrm{DA}} \rightarrow \mathrm{NAcL}$ at cells receive input from $\mathrm{VTA}^{\mathrm{DA}} \rightarrow \mathrm{mPFC}$ cells, then RABV could spread to $533 \mathrm{VTA}^{\mathrm{DA}} \rightarrow \mathrm{mPFC}$ cells and subsequently, their inputs. This interconnectivity thus has the potential

534 to degrade pathway-specific connectivity. Our TRIO strategy was designed to avoid this potential

535 degradation by injection of a third virus, which we chose to be the canine adenovirus (CAV-2)

536 (Beier et al., 2015; Lerner et al., 2015; Schwarz et al., 2015) and later $A A V_{\text {retro }}$ or herpes simplex

537 virus (Ren et al., 2018). We employed a dual recombinase AND-gate where cell types were

538 defined by Cre expression and outputs defined by expression of Cre-dependent Flp recombinase,

539 delivered via CAV-2. Our results from this study suggest that the AND-gate TRIO strategy is

540 required for maintaining pathway specificity in input-output analysis.

\section{Distributed serotonergic inputs to VTA ${ }^{\mathrm{DA}}$ cells}

543 In addition to DA, serotonin plays a key role in the modulation of VTA ${ }^{\mathrm{DA}}$ cells. Multiple types of serotonin receptors are expressed in the VTA, including 5-HT1B, 5-HT2A, and 5-HT2C (Bubar \& Cunningham, 2007; Doherty \& Pickel, 2000; Pazos \& Palacios, 1985; Waeber et al., 1989). Application of serotonin to the VTA depolarizes dopamine neurons, likely through 5-HT2A receptors (Paolucci et al., 2003; Pessia et al., 1994). Studies using classical methods including horseradish peroxidase and radiolabeling found that the VTA receives inputs from brain regions that include serotonergic neurons, including most prominently the DR (Azmitia \& Segal, 1978; Hervé et al., 1987; Parent et al., 1981; Phillipson, 1979). Our results using one-step RABV reinforce these previous findings. However, we also found that DA neurons receive direct innervation from serotonergic neurons located in several other regions, including the B3, B5, B6, $\mathrm{B} 8$, and $\mathrm{B} 9$ regions. The sum of these inputs equates to approximately $1.5 \%$ of total inputs to $V_{T A}{ }^{D A}$ cells. While this may seem insubstantial, serotonergic inputs from the DR only represent about $3.7 \%$ of total inputs to VTA ${ }^{\mathrm{DA}}$ cells, yet these provide a powerful input to VTA ${ }^{\mathrm{DA}}$ cells that

556 influences reward behavior by triggering DA release in the NAc (Liu et al., 2014; McDevitt et al., 557 2014; Qi et al., 2014; Wang et al., 2019). It is also possible that this underrepresents the total 558 serotonergic influence on VTA ${ }^{\mathrm{DA}}$ cells, as serotonin signaling, like DA, is thought to occur in part 559 by volume transmission, therefore likely reducing the efficiency of RABV labeling. Notably, many 
serotonergic neurons in the DR also co-release glutamate, which contributes to the rewarding phenotype of stimulation. It is possible that serotonergic neurons in other brain sites may corelease other neurotransmitters as well, which may work in concert with serotonin to modulate the activity and function of VTA ${ }^{\mathrm{DA}}$ cells.

\section{Practical concerns about local labeling without $T C^{66 T}$}

566 The use of $T C^{66 T}$ enables analysis of inputs near to the site of injection. This approach is required 567 due to high levels of non-Cre-mediated, TVA-facilitated infection of EnvA-pseudotyped RABV.

568 This highlights the issue that investigators regularly analyze brain regions near the injection site without performing the proper controls. The issue is not with the particular AAV vectors used for RABV mapping, but are representative of problems inherent in all standard AAV vectors. While FLEx $^{\operatorname{loxP}}$ and DIO cassettes are typically sufficient to silence expression of fluorescent reporter genes and functional effectors such as DREADDs or optogenetic proteins, low levels of non-Cremediated expression are a problem when only a small amount of gene product is required, such as in the case of recombinase proteins or viral receptors (Botterill et al., 2021; Miyamichi et al., 2013). In this case, even a low level of expression is sufficient to degrade specificity. Note that in control experiments where Cre-dependent TVA and RABV-G were introduced in non-Creexpressing mice, we observed many fewer RABV-labeled cells located at a distance from the injection site. These results suggest that the leak of TVA was sufficient to mediate a substantial amount of infection of RABV locally, as well as some inputs located at a distance from the injection site. Given that we did not observe long-distance inputs labeled when AAV was not injected into the ventral midbrain, it is likely that labeled cells located at a distance from the midbrain were due to retrograde uptake of the TVA-expressing virus, and non-Cre-mediated expression of TVA from these inputs.

Given that nearly all RABV mapping experiments use wild-type TVA and many analyze virallylabeled cells near the injection site, it is important that the issue of non-recombinase-mediated, TVA-facilitated infection of EnvA-pseudotyped RABV is recognized by the community. As with other techniques, application of proper controls should be required for proper interpretation of RABV mapping experiments. We hope that our study serves as an exemplar for the application of such controls and the kinds of questions that can be investigated using the appropriate combination of viral vectors, as we used here to elucidate the nature of inhibitory and neuromodulatory inputs to DA cells in the VTA. 
Acknowledgements:

595 We would like to thank Pieter Derdeyn for assistance with UMAP analysis, and May Hui for 596 assistance with figure production. This work was funded by NIH DP2-AG067666, R00-D041445, 597 R01-DA054374, TRDRP T31KT1437, and T31IP1426, One Mind OM-5596678, Alzheimer's 598 Association AARG-NTF-20-685694, New Vision Research CCAD2020-002, and ADPA APDA5589562.

\section{Competing interests:}

602 The author has no competing interests to report.

\section{MATERIALS AND METHODS:}

605 Mice

606 DAT-Cre mice were obtained from the Jackson Laboratories (Backman et al., 2006). Mice were 607 housed on a $12 \mathrm{hr}$ light/dark cycle with food and water ad libitum. Viral vectors were prepared as 608 previously described (Schwarz et al., 2015). All procedures followed animal care and biosafety 609 guidelines approved by the University of California, Irvine's Administrative Panel on Laboratory 610 Animal Care and Administrative Panel of Biosafety. Both males and females were used in all 611 experiments in approximately equal proportions.

\section{Viral tracing}

614 One-step RABV input mapping was performed as previously described, with the substitution of $615 A A V_{5}-C A G-F L E x^{10 x P}-T C^{66 T}$ in place of $A A V_{5}-C A G-F L E x^{\text {loxP }}-T C^{B}$ (Beier et al., 2015). Briefly, $100 \mathrm{~nL}$ 616 of a $1: 1$ volume mixture of $A A V_{5}-C A G-F L E x^{10 x P}-T C^{66 T}$ and $A A V_{8}-C A G-F L E x^{10 x P}-R A B V-G$ was

617 injected into the VTA of 6-week-old mice. Two weeks later, EnvA-pseudotyped, GFP-expressing 618 RABV was injected into the VTA. Mice were allowed to recover for 5 days to enable viral spread, 619 after which time experiments were terminated.

621 The titers of viruses, based on quantitative PCR analysis, were as follows:

$622 A A V_{5}-C A G-F L E x^{10 x P}-T C^{66 T}, 2.4 \times 10^{13}$ genome copies $(\mathrm{gc}) / \mathrm{mL}$

$623 A A V_{8}-C A G-F L E x^{\text {loxP }}-R A B V-G, 1.0 \times 10^{12} \mathrm{gc} / \mathrm{mL}$.

625 The titer of EnvA-pseudotyped RABV was estimated to be $5.0 \times 10^{8}$ colony forming units (cfu)/mL 626 based on serial dilutions of the virus stock followed by infection of the 293-TVA800 cell line. 


\section{Quantification of signal to noise ratio of RABV input mapping}

629 In order to quantify the signal to noise ratio in $T C^{66 T}$ vs $T C^{B}$-mediated tracing, we considered 1)

630 the number of neurons labeled local to the injection site in Cre- animals, which was considered

631 the local background, 2) both the long-range and local inputs. We assumed that local inputs would

632 comprise $46 \%$ of the total inputs for both $T C^{B}$ and $T C^{66 T}$-mediated tracing; therefore we multiplied

633 the total inputs for $T C^{B}$ experiments by a constant factor (1.87) that was equal to the total inputs

634 divided by the long-range inputs from $T C^{66 T}$ tracing experiments. This resulted in an average total

635 for $T C^{B}$-mediated tracing of 40,955 inputs.

637 For $T C^{B}$ mapping experiments, we counted on average 3,183 local background cells and 638 estimated a total of 40,955 RABV-labeled inputs. Therefore, this resulted in a signal/noise ratio of $63940,955 / 3,183=12.9$. For $T C^{66 T}$ mapping experiments, we counted on average 2.67 local 640 background cells and an average of 23,170 inputs. This yields a signal: noise ratio of 8,710 .

\section{Immunohistochemistry}

643 Animals were transcardially perfused with phosphate buffered saline (PBS) followed by $4 \%$ 644 formaldehyde. Brains were dissected, post-fixed in 4\% formaldehyde for 12-24 hours, and placed in 30\% sucrose for $24-48$ hours. They were then embedded in Tissue Freezing media and stored in a $-80^{\circ} \mathrm{C}$ freezer until sectioning. For RABV tracing analysis, consecutive $60-\mu \mathrm{m}$ coronal sections were collected onto Superfrost Plus slides and stained for NeuroTrace Blue (NTB, Invitrogen). For NTB staining, slides were washed 1x5 min in PBS, 2x10 min in PBS with 0.3\% Triton X-100 (PBST), incubated for 2-3 hours at RT in (1:500) NTB in PBST, washed $1 \times 20$ min with PBST and 1x5min with PBS. Sections were additionally stained with DAPI $(1: 10,000$ of 5 $\mathrm{mg} / \mathrm{mL}$, Sigma-Aldrich), which was included in the last PBST wash of NTB staining. Whole slides were then imaged with a 4x objective using an IX83 Olympus microscope.

For starter cell identification, sections were unmounted after slide scanning, blocked in PBST and $10 \%$ NGS for 2-3 hours at room temperature, and incubated in rat anti-mCherry antibody (1:2000, Life Sciences) and rabbit anti-TH antibody (1:1000, Millipore) at $4^{\circ} \mathrm{C}$ for four nights. After primary antibody staining, sections were washed 3x10 min in PBST, and secondary antibodies (donkey anti-rat Cy3 and donkey anti-rabbit 647, Jackson ImmunoResearch) were applied for two nights at $4^{\circ} \mathrm{C}$, followed by $3 \times 10 \mathrm{~min}$ washes in PBST and remounting. Confocal z-stacks were acquired using a $20 x$ objective on a Zeiss LSM 780 confocal microscope. 


\section{Fluorescent In Situ Hybridization (FISH)}

663 We performed FISH experiments as previously described (Weissbourd et al., 2014). To make ISH 664 probes, DNA fragments of 400-1000 bp containing the coding or untranslated region sequences 665 were amplified by PCR from mouse whole brain cDNA (Zyagen) and subcloned into pCR-BluntII666 topo vector (Life Technologies, cat\# K2800-20). The T3 RNA polymerase recognition site 667 (AATTAACCCTCACTAAAGGG) was added to the 3'-end of the PCR product. Plasmids were 668 then amplified, the insert removed via EcoRI (New England Biolabs, cat \#R0101L) digest, and 669 purified using a PCR purification kit (QIAGEN, cat \#28104). 500-1000 ng of the DNA fragment 670 was then used for in vitro transcription by using DIG RNA labeling mix (cat \#11277073910) and 671 T3 RNA polymerase (cat \#11031163001) according to the manufacturer's instruction (Roche 672 Applied Science). After DNase I (Roche Applied Science, cat \#04716728001) treatment for 30 673 min at $37^{\circ} \mathrm{C}$, the RNA probe was purified by ProbeQuant G-50 Columns (GE Healthcare, cat\# 28674 9034-08) according to the manufacturer's instructions. 60- $\mu \mathrm{m}$ consecutive sections were collected 675 onto Superfrost slides (no-coating, Fisher Scientific, cat \#22-034-980), dried, and stored at $-80^{\circ} \mathrm{C}$ 676 until use. Specific slides were then thawed and viewed on an Olympus compound fluorescence 677 microscope, and the sections containing regions of interest were recorded. Those sections were then floated off using PBS into wells of a 24-well plate. The sections were fixed for $15 \mathrm{~min}$ in $4 \%$ formaldehyde in PBS at room temperature, rinsed with PBS, and incubated with $7 \mu \mathrm{g} / \mathrm{ml}$ Proteinase K (Life Technologies, cat \#25530-049) in $10 \mathrm{mM}$ Tris-Cl, pH 7.4, $1 \mathrm{mM}$ EDTA for 10 min at $37^{\circ} \mathrm{C}$. After fixing again with $4 \%$ formaldehyde in PBS for $10 \mathrm{~min}$ and rinsing with PBS, the sections were incubated with $0.25 \%$ acetic anhydride in $0.1 \mathrm{M}$ triethanolamine, $\mathrm{pH} 8.0$, for $15 \mathrm{~min}$ and washed with PBS. Probes were diluted ( 1:1000) with the hybridization buffer $(50 \%$

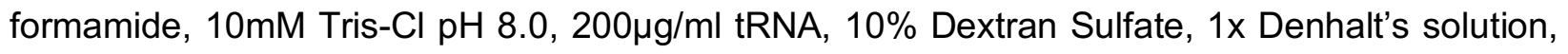
$600 \mathrm{mM} \mathrm{NaCl}, 0.25 \% \mathrm{SDS}$ ), mixed well, preheated at $85^{\circ} \mathrm{C}$ for $5 \mathrm{~min}$, and applied to each well (300-500 $\mu \mathrm{l} /$ well). After $16-20 \mathrm{~h}$ of incubation at $50^{\circ} \mathrm{C}$, the sections were washed, first with $2 \times$ SSC$50 \%$ formamide, then with $2 \times$ SSC, and finally with $0.2 \times$ SSC twice for 20 min at $65^{\circ} \mathrm{C}$. After blocking for $1-2 \mathrm{~h}$ with the $1 \%$ blocking reagent (Roche Applied Science, cat\# 10057177103), sections were incubated with alkaline phosphatase-conjugated anti-DIG antibody (1:1000, Roche Applied Science, cat\# 1093274) and chicken anti-GFP antibodies (1:500; Aves Labs, cat\# GFP-

692 for $15 \mathrm{~min}$ followed by rinsing with the detection buffer $(100 \mathrm{mM}$ Tris-Cl pH8.0, 100mM NaCl, $69310 \mathrm{mM} \mathrm{MgCl}$ ), probe-positive cells were detected by Fast Red TR/Naphthol AS-MX Tablets 694 (Sigma-Aldrich, cat\# F4523). After washing with Roche Wash Buffer three times for 10 min, 695 sections were incubated with FITC-conjugated donkey anti-chicken antibodies (1:200; Jackson 
696 ImmunoResearch) for an additional 1-2h, and washed with PBS three times for 10 min. Finally,

697 the sections were treated with PBS containing 4',6-diamidino-2-phenylindole dihydrochloride

698 (DAPI; Sigma-Aldrich, cat\# D8417) for $20 \mathrm{~min}$ and mounted with cover glass using Fluorogel

699 (Electron Microscopy Sciences, Cat\#17985-10). Sections were imaged by confocal microscopy

700 (Zeiss 780). Images were processed in ImageJ. We used the Cell Counter plugin in FIJI to quantify

701 overlap.

702

\section{Dimensional reduction of RABV input data}

704 Uniform Manifold Approximation and Projection (UMAP) was used as a non-linear dimensional 705 reduction technique on input data. UMAP is optimized for finding local and global structures in 706 high dimensional data. Analyses were performed using the official UMAP library (Mclnnes et al., 707 2018). The fractional counts data were $z$-scored to compare variation in output and input sites 708 across regions with different magnitudes of counts. Z-scored data were dimensionally reduced 709 with UMAP to find clusters of input sites with similar patterns of variation. UMAP parameters were 710 tuned manually to optimize stability of clusters.

Analysis of fluorescent axonal labeling from Allen Mouse Brain Connectivity Atlas

713 Analysis was performed as previously described (Beier et al., 2019). Data from three separate 714 injections for each local VTA ${ }^{\mathrm{DA}}$ input site were analyzed. Experiments had to show GFP labeling 715 in the VTA. For each experiment, three images of the VTA, each spaced approximately two 716 sections apart, were captured at screen resolution. In ImageJ, using the line tool at a thickness of 717100 , a line was drawn from the midline to the end of the medial lemniscus running ventro-lateral 718 through the VTA, and the gray value (a metric of axon coverage) was obtained as a function of 719 distance from the midline. To normalize these values, data were run through custom MATLAB 720 code to segment data into 100 bins and normalized to the maximum intensity value for that image.

721 The three normalized values were then averaged for each brain, and the data from three separate

722 brains then were averaged into one condition and plotted as a percentage across the medial723 lateral axis.

\section{Brain Region Abbreviation List}

726 Abbreviations for brain regions made throughout the paper are listed below, in alphabetical order:

727 Long-range inputs:

728 Ant. Ctx./cortex - anterior cortex

729 BNST - bed nucleus of the stria terminalis 
730 CeA - central amygdala

731 DCN - deep cerebellar nucleus

732 DR - dorsal raphe

733 DStr - dorsal striatum

734 EAM - extended amygdala

735 EP - entopeduncular nucleus (GPi)

736 GP - globus pallidus (GPe)

737 LDT - laterodorsal tegmentum

738 LH - lateral hypothalamus

$739 \mathrm{LHb}$ - lateral habenula

$740 \mathrm{MHb}-$ medial habenula

741 NAcCore- nucleus accumbens, core

742 NAcMed - nucleus accumbens, medial shell

743 NAcLat - nucleus accumbens, lateral shell

744 PBN - parabrachial nucleus

745 PO - pre-optic area

$746 \mathrm{PVH}$ - paraventricular hypothalamus

747 VP - ventral pallidum

748 VTA - ventral tegmental area

749 ZI - zona incerta

750

751 Local inputs:

752 ATg - Anterior Tegmental Nucleus

753 DLL - Dorsal Nucleus of the Lateral Lemniscus

754 IC - Inferior Colliculus

755 InC - Interstitial Nucleus of Cajal

756 IPN - Interpeduncular Nucleus

757 MiTg - Microcellular Tegmental Nucleus

758 MnR - Median Raphe

$759 \mathrm{mRT}$ - Midbrain Reticular Nucleus

760 MM - Mammillary Nucleus

761 MPL - Medial Paralemniscal Nucleus

762 PAG - Periaqueductal Gray

763 PMnR - Paramedian Raphe Nucleus 
764 PnC - Pontine Reticular Nucleus, Caudal Part

765 PnO - Pontine Reticular Nucleus, Oral Part

766 PPTg - Pedunculopontine Tegmental Nucleus

767 PSth - Parasubthalamic Nucleus

768 PT - Pretectal Area

$769 \mathrm{RI}$ - Rostral Interstitial Nucleus of Medial Longitudinal Fasciculus

770 Rli - Rostral Linear Nucleus Raphe

$771 \mathrm{RMg}$ - Raphe Magnus

772 RPC - Red Nucleus, Parvocellular Part

773 RRF - Retrorubal Field

774 RtTg - Reticulotegmental Nucleus

775 SC - Superior Colliculus

776 SNc - Substantia Nigra Pars Compacta

777 SNI - Substantia Nigra Pars Lateralis

778 SNr - Substantia Nigra Pars Reticulata

779 SPTg - Subpeduncular Tegmental Nucleus

780 SubB - Sub-Brachial Nucleus

781 SuM - Supramammillary Nucleus

782 SuMx-Supramammillary decussation

783 VTA IF - Ventral Tegmental Area, Interfascicular Nucleus

784 VTA PBP- Ventral Tegmental Area, Parabrachial Pigmented Nucleus

785 VTA PN- Ventral Tegmental Area, Paranigral Nucleus

786 VTg - Ventral Tegmental Nucleus

\section{REFERENCES CITED:}

Alsteens, D., Newton, R., Schubert, R., Martinez-Martin, D., Delguste, M., Roska, B., \& Müller, D. J. (2017). Nanomechanical mapping of first binding steps of a virus to animal cells. Nature Nanotechnology, 12(2), 177-183. https://doi.org/10.1038/nnano.2016.228

Azmitia, E. C., \& Segal, M. (1978). An autoradiographic analysis of the differential ascending projections of the dorsal and median raphe nuclei in the rat. Journal of Comparative Neurology, 179(3), 641-667. https://doi.org/10.1002/cne.901790311

Backman, C. M., Malik, N., Zhang, Y., Shan, L., Grinberg, A., Hoffer, B. J., Westphal, H., \& Tomac, A. C. (2006). Characterization of a mouse strain expressing Cre recombinase from the 3' untranslated region of the dopamine transporter locus. Genesis (New York, N.Y. : 2000), 
44(8), 383-390. https://doi.org/10.1002/dvg.20228

Bayer, V. E., \& Pickel, V. M. (1990). Ultrastructural localization of tyrosine hydroxylase in the rat ventral tegmental area: Relationship between immunolabeling density and neuronal associations. Journal of Neuroscience, 10(9), 2996-3013. https://doi.org/10.1523/jneurosci.10-09-02996.1990

Beckstead, M. J., Grandy, D. K., Wickman, K., \& Williams, J. T. (2004). Vesicular dopamine release elicits an inhibitory postsynaptic current in midbrain dopamine neurons. Neuron, 42(6), 939-946. https://doi.org/10.1016/j.neuron.2004.05.019

Beier, K. T., Gao, X. J., Xie, S., DeLoach, K. E., Malenka, R. C., \& Luo, L. (2019). Topological Organization of Ventral Tegmental Area Connectivity Revealed by Viral-Genetic Dissection of Input-Output Relations. Cell Reports, 26(1), 159-167.e6. https://doi.org/10.1016/j.celrep.2018.12.040

Beier, K. T., Steinberg, E. E., Deloach, K. E., Xie, S., Miyamichi, K., Schwarz, L., Gao, X. J., Kremer, E. J., Malenka, R. C., \& Luo, L. (2015). Circuit Architecture of VTA Dopamine Neurons Revealed by Systematic Input-Output Mapping. Cell, 162(3), 622-634. https://doi.org/10.1016/j.cell.2015.07.015

Björklund, A., \& Dunnett, S. B. (2007). Dopamine neuron systems in the brain: an update. Trends in Neurosciences, 30(5), 194-202. https://doi.org/10.1016/j.tins.2007.03.006

Botterill, J. J., Khlaifia, A., Walters, B. J., Scharfman, H. E., Arruda-carvalho, M., \& Brimble, M. A. (2021). Off-Target Expression of Cre-Dependent Adeno- Associated Viruses in Wild-Type C57BL / 6J Mice. 21(December), 1-16.

Bouarab, C., Thompson, B., \& Polter, A. M. (2019). VTA GABA Neurons at the Interface of Stress and Reward. Frontiers in Neural Circuits, 13(December), 1-12. https://doi.org/10.3389/fncir.2019.00078

Bromberg-Martin, E. S., Matsumoto, M., \& Hikosaka, O. (2010). Dopamine in Motivational Control: Rewarding, Aversive, and Alerting. Neuron, 68(5), 815-834. https://doi.org/10.1016/j.neuron.2010.11.022

Bubar, M. J., \& Cunningham, K. A. (2007). Distribution of serotonin 5-HT2C receptors in the ventral tegmental area. Neuroscience, 146(1), 286-297. https://doi.org/10.1016/j.neuroscience.2006.12.071

Cohen, J. Y., Haesler, S., Vong, L., Lowell, B. B., \& Uchida, N. (2012). Neuron-type-specific signals for reward and punishment in the ventral tegmental area. Nature, 482(7383), 85-88. https://doi.org/10.1038/nature10754

Condon, A. F., Robinson, B. G., Asad, N., Dore, T. M., Tian, L., \& Williams, J. T. (2021). The 
residence of synaptically released dopamine on D2 autoreceptors. Cell Reports, 36(5), 109465. https://doi.org/10.1016/j.celrep.2021.109465

Derdeyn, P., Hui, M., Macchia, D., \& Beier, K. (2022). Uncovering the Connectivity Logic of the Ventral Tegmental Area. Frontiers in Neural Circuits, in press.

Dobi, A., Margolis, E. B., Wang, H. L., Harvey, B. K., \& Morales, M. (2010). Glutamatergic and

Ford, C. P., Phillips, P. E. M., \& Williams, J. T. (2009). The time course of dopamine transmission nonglutamatergic neurons of the ventral tegmental area establish local synaptic contacts with dopaminergic and nondopaminergic neurons. Journal of Neuroscience, 30(1), 218-229. https://doi.org/10.1523/JNEUROSCI.3884-09.2010

Doherty, M. D., \& Pickel, V. M. (2000). Ultrastructural localization of the serotonin 2A receptor in dopaminergic neurons in the ventral tegmental area. Brain Research, 864(2), 176-185. https://doi.org/10.1016/S0006-8993(00)02062-X

Faget, L., Osakada, F., Duan, J., Ressler, R., Johnson, A. B., Proudfoot, J. A., Yoo, J. H., Callaway, E. M., \& Hnasko, T. S. (2016). Afferent Inputs to Neurotransmitter-Defined Cell Types in the Ventral Tegmental Area. Cell Reports, 15(12), 2796-2808. in the ventral tegmental area. Journal of Neuroscience, 29(42), 13344-13352. https://doi.org/10.1523/JNEUROSCI.3546-09.2009

Gantz, S. C., Bunzow, J. R., \& Williams, J. T. (2013). Spontaneous inhibitory synaptic currents mediated by a $\mathrm{g}$ protein-coupled receptor. Neuron, 78(5), 807-812. https://doi.org/10.1016/j.neuron.2013.04.013

Geisler, S., Derst, C., Veh, R. W., \& Zahm, D. S. (2007). Glutamatergic afferents of the ventral tegmental area in the rat. Journal of Neuroscience, 27(21), 5730-5743. https://doi.org/10.1523/JNEUROSCI.0012-07.2007

Geisler, S. \& Zahm, D. S. (2005). Afferents of the ventral tegmental area in the rat-anatomical substratum for integrative functions. Journal of Comparative Neurology, 490(3), 270-294. https://doi.org/10.1002/cne.20668

Groves, P.M., \& Linder, J.C. (1983). Dendro-dendritic synapses in substantia nigra: descriptions based on analysis of serial sections. Exp Brain Res, 49(2), 209-217.

Hajdu, F., Hassler, R., \& Bak, I. J. (1973). Electron microscopic study of the substantia nigra and

864 Hensler, J. G., Artigas, F., Bortolozzi, A., Daws, L. C., De Deurwaerdère, P., Milan, L., Navailles, 865 S., \& Koek, W. (2013). Catecholamine/serotonin interactions. Systems thinking for brain 
866

function and disease. In Advances in Pharmacology (1st ed., Vol. 68). Elsevier Inc. https://doi.org/10.1016/B978-0-12-411512-5.00009-9

Hervé, D., Pickel, V. M., Joh, T. H., \& Beaudet, A. (1987). Serotonin axon terminals in the ventral tegmental area of the rat: fine structure and synaptic input to dopaminergic neurons. Brain Research, 435(1-2), 71-83. https://doi.org/10.1016/0006-8993(87)91588-5

Jhou, T. (2005). Neural mechanisms of freezing and passive aversive behaviors. Journal of Comparative Neurology, 493(1), 111-114. https://doi.org/10.1002/cne.20734

Jhou, T. C., Fields, H. L., Baxter, M. G., Saper, C. B., \& Holland, P. C. (2009a). The Rostromedial Tegmental Nucleus (RMTg), a GABAergic Afferent to Midbrain Dopamine Neurons, Encodes Aversive Stimuli and Inhibits Motor Responses. Neuron, 61(5), 786-800. https://doi.org/10.1016/j.neuron.2009.02.001

Jhou, T. C., Geisler, S., Marinelli, M., Degarmo, B. A., \& Zahm, D. S. (2009b). The mesopontine rostromedial tegmental nucleus: A structure targeted by the lateral habenula that projects to the ventral tegmental area of Tsai and substantia nigra compacta. Journal of Comparative Neurology, 513(6), 566-596. https://doi.org/10.1002/cne.21891

Kaufling, J., Veinante, P., Pawlowski, S. A., Freund-Mercier, M. J., \& Barrot, M. (2009). Afferents to the GABAergic tail of the ventral tegmental area in the rat. Journal of Comparative Neurology, 513(6), 597-621. https://doi.org/10.1002/cne.21983

Kim, C. K., Yang, S. J., Pichamoorthy, N., Young, N. P., Kauvar, I., Jennings, J. H., Lerner, T. N., Berndt, A., Lee, S. Y., Ramakrishnan, C., Davidson, T. J., Inoue, M., Bito, H., \& Deisseroth, K. (2016). Simultaneous fast measurement of circuit dynamics at multiple sites across the mammalian brain. Nature Methods, 13(4), 325-328. https://doi.org/10.1038/nmeth.3770

Lammel, S., Hetzel, A., Häckel, O., Jones, I., Liss, B., \& Roeper, J. (2008). Unique Properties of Mesoprefrontal Neurons within a Dual Mesocorticolimbic Dopamine System. Neuron, 57(5), 760-773. https://doi.org/10.1016/j.neuron.2008.01.022

Lammel, S., Ion, D. I., Roeper, J., \& Malenka, R. C. (2011). Report Projection-Specific Modulation of Dopamine Neuron Synapses by Aversive and Rewarding Stimuli. Neuron, 70(5), 855-862. https://doi.org/10.1016/j.neuron.2011.03.025

Lammel, S., Lim, B. K., Ran, C., Huang, K. W., Betley, M. J., Tye, K. M., Deisseroth, K., \& Malenka, R. C. (2012). Input-specific control of reward and aversion in the ventral tegmental area. Nature, 491(7423), 212-217. https://doi.org/10.1038/nature11527

Lerner, T. N., Shilyansky, C., Davidson, T. J., Evans, K. E., Beier, K. T., Zalocusky, K. A., Crow, A. K., Malenka, R. C., Luo, L., Tomer, R., \& Deisseroth, K. (2015). Intact-Brain Analyses Reveal Distinct Information Carried by SNc Dopamine Subcircuits. Cell, 162(3), 635-647. 
https://doi.org/10.1016/j.cell.2015.07.014

Liu, Z., Zhou, J., Li, Y., Hu, F., Lu, Y., Ma, M., Feng, Q., Zhang, J. en, Wang, D., Zeng, J., Bao, J., Kim, J. Y., Chen, Z. F., EIMestikawy, S., \& Luo, M. (2014). Dorsal raphe neurons signal reward through 5-HT and glutamate. Neuron, 81(6), 1360-1374. https://doi.org/10.1016/j.neuron.2014.02.010

McDevitt, R. A., Tiran-Cappello, A., Shen, H., Balderas, I., Britt, J. P., Marino, R. A. M., Chung, S. L., Richie, C. T., Harvey, B. K., \& Bonci, A. (2014). Serotonergic versus nonserotonergic dorsal raphe projection neurons: Differential participation in reward circuitry. Cell Reports, 8(6), 1857-1869. https://doi.org/10.1016/j.celrep.2014.08.037

Mclnnes, L., Healy, J., Saul, N., \& Großberger, L. (2018). UMAP: Uniform Manifold Approximation and Projection. Journal of https://doi.org/10.21105/JOSS.00861

Menegas, W., Bergan, J. F., Ogawa, S. K., Isogai, Y., Venkataraju, K. U., Osten, P., Uchida, N., \& Watabe-Uchida, M. (2015). Dopamine neurons projecting to the posterior striatum form an anatomically distinct subclass.

ELife, 4(AUGUST2015), $1-30$. https://doi.org/10.7554/eLife.10032

Miyamichi, K., Shlomai-Fuchs, Y., Shu, M., Weissbourd, B. C., Luo, L., \& Mizrahi, A. (2013). Dissecting local circuits: Parvalbumin interneurons underlie broad feedback control of olfactory bulb output. Neuron, $\quad$ 80(5), 1232-1245. https://doi.org/10.1016/j.neuron.2013.08.027

Paolucci, E., Berretta, N., Tozzi, A., Bernardi, G., \& Mercuri, N. B. (2003). Depression of mGluRmediated IPSCs by 5-HT in dopamine neurons of the rat substantia nigra pars compacta. European Journal of Neuroscience, 18(10), 2743-2750. https://doi.org/10.1111/j.14609568.2003.03015.x

Parent, A., Descarries, L., \& Beaudet, A. (1981). Organization of ascending serotonin systems in the adult rat brain. A radioautographic study after intraventricular administration of [3h]5hydroxytryptamine. Neuroscience, 6(2). https://doi.org/10.1016/0306-4522(81)90050-6

Pazos, A., \& Palacios, J. M. (1985). Quantitative autoradiographic mapping of serotonin receptors in the rat brain. I. Serotonin-1 receptors. Brain Research, 346(2), 205-230. https://doi.org/10.1016/0006-8993(85)90856-X

Pessia, M., Jiang, Z. G., Alan North, R., \& Johnson, S. W. (1994). Actions of 5-hydroxytryptamine on ventral tegmental area neurons of the rat in vitro. Brain Research, 654(2), 324-330. https://doi.org/10.1016/0006-8993(94)90495-2

Phillipson, O. T. (1979). Afferent projections to the ventral tegmental area of Tsai and 
interfascicular nucleus: A horseradish peroxidase study in the rat. Journal of Comparative Neurology, 187(1), 117-143. https://doi.org/10.1002/cne.901870108

Qi, J., Zhang, S., Wang, H. L., Wang, H., De Jesus Aceves Buendia, J., Hoffman, A. F., Lupica,

Rong, L., Gendron, K., Strohl, B., Shenoy, R., Wool-Lewis, R. J., \& Bates, P. (1998).

Sesack, S. R., \& Grace, A. A. (2010). Cortico-basal ganglia reward network: Microcircuitry. Neuropsychopharmacology, 35(1), 27-47. https://doi.org/10.1038/npp.2009.93

Swanson, L. W. (2000). Cerebral hemisphere regulation of motivated behavior11Published on the World Wide Web on 2 November 2000. Brain Research, 886(1-2), 113-164. https://doi.org/10.1016/s0006-8993(00)02905-x

Tan, K. R., Yvon, C., Turiault, M., Mirzabekov, J. J., Doehner, J., Labouèbe, G., Deisseroth, K.,

Wall, N. R., De La Parra, M., Callaway, E. M., \& Kreitzer, A. C. (2013). Differential innervation of Tye, K. M., \& Lüscher, C. (2012). GABA Neurons of the VTA Drive Conditioned Place Aversion. Neuron, 73(6), 1173-1183. https://doi.org/10.1016/j.neuron.2012.02.015

Waeber, C., Dietl, M., Hoyer, D., \& Palacios, J. (1989). 5.HT1 receptors in the vertebrate brain. Regional distribution examined by autoradiography. Naunyn Schmiedebergs Arch Pharmacol ., 340(5), 486-494. direct- and indirect-pathway striatal projection neurons. Neuron, 79(2), 347-360. https://doi.org/10.1016/j.neuron.2013.05.014

Wang, H. L., Zhang, S., Qi, J., Wang, H., Cachope, R., Mejias-Aponte, C. A., Gomez, J. A., MateoSemidey, G. E., Beaudoin, G. M. J., Paladini, C. A., Cheer, J. F., \& Morales, M. (2019). 

Synapses on VTA Mesoaccumbens Dopamine Neurons. Cell Reports, 26(5), 1128-1142.e7. https://doi.org/10.1016/j.celrep.2019.01.014

Watabe-Uchida, M., Zhu, L., Ogawa, S. K., Vamanrao, A., \& Uchida, N. (2012). Whole-Brain Mapping of Direct Inputs to Midbrain Dopamine Neurons. Neuron, 74(5), 858-873. https://doi.org/10.1016/j.neuron.2012.03.017

974 Weissbourd, B., Ren, J., DeLoach, K. E., Guenthner, C. J., Miyamichi, K., \& Luo, L. (2014).

975 Presynaptic Partners of Dorsal Raphe Serotonergic and GABAergic Neurons. Neuron, 83(3), 645-662. https://doi.org/10.1016/j.neuron.2014.06.024

Wickersham, I. R., Lyon, D. C., Barnard, R. J. O., Mori, T., Finke, S., Conzelmann, K.-K., Young, J. A. T., \& Callaway, E. M. (2007). Monosynaptic restriction of transsynaptic tracing from

Wilson, C. J., Groves, P. M., \& Fifková, E. (1977). Monoaminergic synapses, including dendrodendritic synapses in the rat substantia nigra. Experimental Brain Research, 30(2-3), 161174. https://doi.org/10.1007/BF00237248

984 Wise, R. A. (2004). Dopamine, learning and motivation. Nature Reviews. Neuroscience, 5(6), 483-494. https://doi.org/10.1038/nrn1406

Zahm, D. S., Cheng, A. Y., Lee, T. J., Ghobadi, C. W., Schwartz, Z. M., Geisler, S., Parsely, K. P., Gruber, C., \& Veh, R. W. (2011). Inputs to the midbrain dopaminergic complex in the rat, with emphasis on extended amygdala-recipient sectors. The Journal of Comparative Neurology, 519(16), 3159-3188. https://doi.org/10.1002/CNE.22670 